\title{
Configuration spaces of thick particles on a metric graph
}

\author{
KENNETH DEELEY
}

\begin{abstract}
We study the topology of configuration spaces $F_{r}(\Gamma, 2)$ of two thick particles (robots) of radius $r>0$ moving on a metric graph $\Gamma$. As the size of the robots increases, the topology of $F_{r}(\Gamma, 2)$ varies. Given $\Gamma$ and $r$, we provide an algorithm for computing the number of path components of $F_{r}(\Gamma, 2)$. Using our main tool of PL Morse-Bott theory, we show that there are finitely many critical values of $r$ where the homotopy type of $F_{r}(\Gamma, 2)$ changes. We study the transition across a critical value $R \in(a, b)$ by computing the ranks of the relative homology groups of $\left(F_{a}(\Gamma, 2), F_{b}(\Gamma, 2)\right)$.
\end{abstract}

55R80, 57Q05; 57M15

\section{Introduction}

In this article we study the topology of configuration spaces of two thick particles (robots) moving on a metric graph. This work is motivated by problems arising in topological robotics; see Farber's book [9] for an introduction. Classical configuration spaces of graphs arise naturally in this context. For example, consider the engineering problem of safely organising multiple Automated Guided Vehicles (AGVs) moving without collisions on a fixed guidepath network. This problem has been studied by Abrams [1], Abrams and Ghrist [2], Ghrist [11; 12] and Ghrist and Koditschek [13; 14]. The natural model assumes that the guidepath network is a graph $\Gamma$; the space of all collision-free positions of $n$ AGVs is the classical $n$-point configuration space

$$
F(\Gamma, n):=\left\{\left(x_{1}, \ldots, x_{n}\right) \in \Gamma^{n}: x_{i} \neq x_{j} \text { for } i \neq j\right\} .
$$

In [11] it is proved that $F(\Gamma, n)$ deformation retracts onto a finite $\mathrm{CW}$ complex of dimension dependent only on $\Gamma$ (and not on $n$ ). A similar result was proved independently by Świątkowski in [16]. The configuration space of $n$ points on a tree $T$ was studied by Farber [8], who describes an explicit motion planning algorithm in $F(T, n)$ and computes the topological complexity of $F(T, n)$ (see Chapter 4 of [9] for definitions).

Many important and interesting results have been obtained for classical two-point configuration spaces of graphs. For example, Farber [8] completely describes the topology of $F(T, 2)$ for a tree $T$. In the case of a planar graph, Barnett and Farber [4] 
describe the free generators of $H_{2}(F(\Gamma, 2), \mathbb{Z})$ and also explicitly describe the cohomology algebra $H^{*}(F(\Gamma, 2), \mathbb{Q})$ for any regular planar graph $\Gamma$; see also Barnett [3]. The topology of $F(\Gamma, 2)$ for nonplanar graphs $\Gamma$ is studied by Farber and Hanbury in [10]. Importantly, Ko and Park [15] have shown that $H_{1}(F(\Gamma, 2))$ is free for any finite connected graph $\Gamma$ and have computed $\operatorname{rk} H_{1}(F(\Gamma, 2))$ explicitly in terms of graph-theoretic invariants.

However, it has been recognised in the literature that viewing the moving objects (robots) as zero-size points is not completely realistic from a modelling stance. For example, in the context of scheduling AGVs in a factory, Abrams and Ghrist [2] acknowledge that "Of course, since the robots are not truly points, and since no control algorithm implementation is of infinite precision, we require that the control path reside outside of a neighborhood of the diagonal $\Delta$ in $\left(\mathbb{R}^{2}\right)^{N}$." In this article we attempt to address such issues by modelling the moving objects as metric balls of positive radius in $\Gamma$ (and not as zero-size points). We emphasise that with this approach, we study a new family of configuration spaces of "thick" (ie positive radius) particles on a metric graph.

Basic definitions and properties We work with a finite, simple, connected graph $\Gamma$ in which the edges are labelled by positive numbers. Such a labelling induces a metric $d$ on $\Gamma$, where $d(x, y)$ is the length $L_{\gamma}$ of a shortest arc $\gamma$ connecting $x$ to $y$. We write $\operatorname{diam}(\Gamma)=\max \{d(x, y): x, y \in \Gamma\}$ for the diameter of $\Gamma$. We use the notation $\mu(v)$ for the number of edges incident to the vertex $v$, and we set $\mu(x)=2$ if $x \in \Gamma$ is not a vertex. A star neighbourhood of $x \in \Gamma$ is an open set containing $x$ and homeomorphic to the wedge sum of $\mu(x)$ copies of $[0,1)$ such that $x$ corresponds to the wedge point 0 under the homeomorphism. For $r>0$, we define the corresponding thick particle configuration space to be

$$
F_{r}(\Gamma, 2):=\{(x, y) \in \Gamma \times \Gamma: d(x, y) \geq 2 r\},
$$

topologised as a subspace of $\Gamma \times \Gamma$. The space $F_{r}(\Gamma, 2)$ models the collision-free motion of two robots of radius $r$ on $\Gamma$, where tangencies between the robots are permitted. By varying $r$, we obtain a family of configuration spaces $\left\{F_{r}(\Gamma, 2)\right\}_{r>0}$. We have $F_{r}(\Gamma, 2) \subset F(\Gamma, 2)$ for all $r$. It is easy to check that $F_{r}(\Gamma, 2)=\varnothing$ if and only if $r>\frac{1}{2} \operatorname{diam}(\Gamma)$.

Main results In Section 2 we give an algorithm for computing the number of path components of $F_{r}(\Gamma, 2)$ given $\Gamma$ and $r$. We use the notation $b_{0}(X)$ for the 0 -th Betti number of a space $X$ (the number of path components of $X$ ). The main result of Section 2 is as follows (see Theorem 2.11). 
Theorem 1.1 Given $\Gamma$ and $r$, we describe an algorithm for constructing a graph $G_{r}$ which embeds into $F_{r}(\Gamma, 2)$ and satisfies $b_{0}\left(F_{r}(\Gamma, 2)\right)=b_{0}\left(G_{r}\right)$.

To illustrate the general complexity of the family $\left\{F_{r}(\Gamma, 2)\right\}_{r>0}$ we refer to Example 2.13, in which Theorem 1.1 shows that $b_{0}\left(F_{r}(\Gamma, 2)\right)$ need not be a monotone (or even unimodal) function of $r$.

In Section 3 we develop our main tool of PL Morse-Bott theory and apply it in Section 5 to obtain information about the homotopy type of the spaces $\left\{F_{r}(\Gamma, 2)\right\}_{r>0}$. The following definition makes precise the notion of a critical value in this context.

Definition 1.2 A number $R>0$ is a regular value of the family $\left\{F_{r}(\Gamma, 2)\right\}_{r>0}$ if there is an open set $U_{R} \subset(0, \infty)$ containing $R$ such that $F_{S}(\Gamma, 2)$ is a deformation retract of $F_{r}(\Gamma, 2)$ for all $r, s \in U_{R}$ with $r \leq s$. A number $R>0$ is a critical value of $\left\{F_{r}(\Gamma, 2)\right\}_{r>0}$ if it is not a regular value.

The main result of Section 5 is as follows (see Theorem 5.1):

Theorem 1.3 There are fewer than $2^{E} \max \{2 Z(Z-1), 1\}$ critical values, where $E$ and $Z$ are the numbers of edges and cycles in $\Gamma$, respectively.

We obtain this statement by expressing each critical value explicitly in terms of metric properties of $\Gamma$ (see Theorem 5.1). In Section 5 we also apply the PL Morse-Bott theory to compute the ranks of the relative homology groups

$$
H_{k}\left(F_{a}(\Gamma, 2), F_{b}(\Gamma, 2) ; \mathbb{Q}\right), \text { for } k=0,1,2 .
$$

Here $(a, b)$ contains a single critical value $R$. Finally, we describe explicit generators for $H_{2}\left(F_{a}(\Gamma, 2), F_{b}(\Gamma, 2) ; \mathbb{Q}\right)$.

Outline In Section 2 we introduce index zero configurations and show that every path component of $F_{r}(\Gamma, 2)$ contains such a configuration. We then construct a graph $G_{r}$ which embeds in $F_{r}(\Gamma, 2)$ such that the inclusion-induced map $\iota_{*}: \pi_{0}\left(G_{r}\right) \rightarrow$ $\pi_{0}\left(F_{r}(\Gamma, 2)\right)$ is a bijection. This enables us to write down an algorithm for computing $b_{0}\left(F_{r}(\Gamma, 2)\right)$ given $\Gamma$ and $r$. We develop a PL Morse-Bott theory for affine polytope complexes in Section 3. This is our main tool for studying the homotopy type of $F_{r}(\Gamma, 2)$. The PL Morse-Bott theory deals with the homotopy type of preimages $X^{c}=f^{-1}((-\infty, c])$ under an affine Morse-Bott function $f: X \rightarrow \mathbb{R}$. We verify in Section 4 that the metric $d$ is an affine Morse-Bott function on $\Gamma \times \Gamma$. In Section 5 we apply the results of Section 3 to $d$ and obtain the statement about critical values and the computation of the relative homology groups as mentioned above (Theorem 1.3 and the statements that follow). Finally in Section 6 we discuss the general case of configuration spaces of $n>2$ thick particles together with some open problems. 
Acknowledgements I thank my PhD supervisor Professor Farber for inspiring and carefully guiding the work contained in this article. I also thank Dr Hanbury for helpful discussions and valuable feedback. I am grateful to an anonymous referee for many insightful comments and suggestions.

\section{Path components}

In this section we develop an algorithm for computing the number of path components of $F_{r}(\Gamma, 2)$ given $\Gamma$ and $r$. We consider $r \in\left(0, \frac{1}{2} \operatorname{diam}(\Gamma)\right]$, since $F_{r}(\Gamma, 2)=\varnothing$ if and only if $r>\frac{1}{2} \operatorname{diam}(\Gamma)$.

\subsection{Index zero configurations}

Definition 2.1 Given distinct points $x, y \in \Gamma$, we define the positive index ind $+(x, y)$ of $(x, y)$ to be the number of branches in a star neighbourhood around $x$ on which $w \mapsto d(w, y)$ increases as we move away from $x$.

Example 2.2 In Figure 1, $\operatorname{ind}_{+}(x, y)=2$ and $\operatorname{ind}_{+}(y, x)=3$.

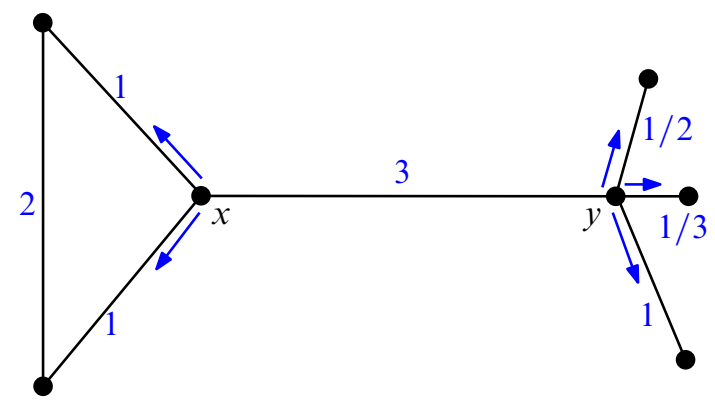

Figure 1: Computing the positive indices $\operatorname{ind}_{+}(x, y), \operatorname{ind}_{+}(y, x)$

Definitions 2.3 A configuration $(x, y) \in F(\Gamma, 2)$ is critical if ind $+(x, y) \neq 1$ and $\operatorname{ind}_{+}(y, x) \neq 1$. A configuration $(x, y) \in F(\Gamma, 2)$ has index zero if $\operatorname{ind}_{+}(x, y)=$ $\operatorname{ind}_{+}(y, x)=0$.

The following result provides an upper bound on $b_{0}\left(F_{r}(\Gamma, 2)\right)$.

Proposition 2.4 For any $r \in\left(0, \frac{1}{2} \operatorname{diam}(\Gamma)\right]$, the number of path components of $F_{r}(\Gamma, 2)$ is bounded above by $b_{0}\left(Z_{r}\right)$, where $Z_{r}$ is the set of index zero configurations $\left(x_{0}, y_{0}\right)$ with $d\left(x_{0}, y_{0}\right) \geq 2 r$. 
Proof Let $P$ be any path component of $F_{r}(\Gamma, 2)$, and fix $(x, y) \in P$. Suppose that $(x, y)$ is not an index zero configuration, and let $\gamma$ be a minimal-length arc from $x$ to $y$.

(a) If $\operatorname{ind}_{+}(x, y)>0$, choose a branch incident to $x$ on which $w \mapsto d(w, y)$ increases as we move away from $x$. Now push $x$ along this branch, stopping if we reach a point $x_{0}$ with ind ${ }_{+}\left(x_{0}, y\right)=0$. By continuing to choose branches in this way if necessary, this procedure terminates with a point $x_{0}$ such that $\operatorname{ind}_{+}\left(x_{0}, y\right)=0$; see Figure 2. Let $\gamma_{1}$ be the arc from $x_{0}$ to $x$ obtained from this procedure. By construction, the concatenation $\gamma_{1} \cdot \gamma$ is a minimal-length arc from $x_{0}$ to $y$.

(b) If $\operatorname{ind}_{+}(x, y)=0$, then we have $\operatorname{ind}_{+}(y, x)>0$, so apply the above procedure to obtain a point $y_{0}$ such that $\operatorname{ind}_{+}\left(y_{0}, x\right)=0$.

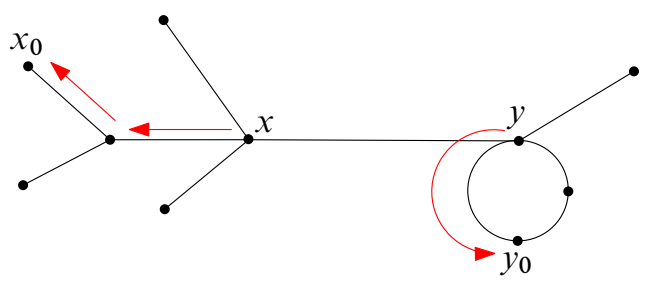

Figure 2: Finding an index zero configuration $\left(x_{0}, y_{0}\right)$

If case (a) applies and if ind $+\left(y, x_{0}\right)>0$, use the same procedure to push $y$ away from $x_{0}$ until we reach a point $y_{0}$ with ind $+\left(y_{0}, x_{0}\right)=0$. Let $\gamma_{2}$ be the arc from $y$ to $y_{0}$ obtained from this procedure. Then by construction, $\left(\gamma_{1} \cdot \gamma\right) \cdot \gamma_{2}$ is a minimallength arc from $x_{0}$ to $y_{0}$. Seeking a contradiction, assume that ind $+\left(x_{0}, y_{0}\right)>0$. Then there exists a branch of a star neighbourhood $U_{x_{0}}$ on which $w \mapsto d\left(w, y_{0}\right)$ increases as we move away from $x_{0}$. Choose a point $x_{0}^{\prime}$ on this branch with $d\left(x_{0}^{\prime}, y_{0}\right)>d\left(x_{0}, y_{0}\right)$. There exists an arc $p$ connecting $x_{0}$ with $y$ and passing through $x_{0}^{\prime}$ which does not pass through $x_{0}$; see Figure 3. Indeed, if such an arc does not exist then we have

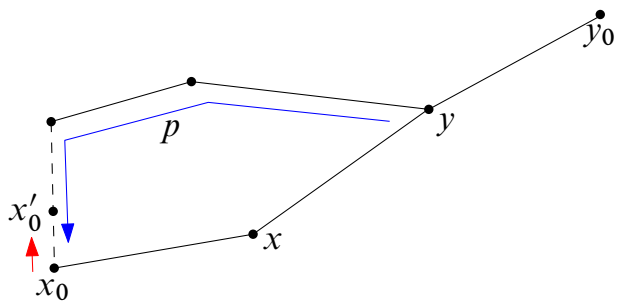

Figure 3: The point $x_{0}^{\prime}$ and the arc $p$ 
ind $_{+}\left(x_{0}, y\right)>0$, a contradiction. We can also assume that $p$ has minimal-length. Indeed, we do not have $L_{p}<L_{\gamma_{1} \cdot \gamma}$, since $\gamma_{1} \cdot \gamma$ is a minimal-length arc from $x_{0}$ to $y$. If no minimal-length arc with the properties of $p$ exists, then $L_{p}>L_{\gamma_{1} \cdot \gamma}$ and we can move $x_{0}$ away from $y$ along $p$ in the direction of $x_{0}^{\prime}$, contradicting $\operatorname{ind}_{+}\left(x_{0}, y\right)=0$. Hence $L_{p}=L_{\gamma_{1} \cdot \gamma}$. In particular, $d\left(x_{0}^{\prime}, y_{0}\right)$ is equal to $L_{\gamma_{2}}+L_{\left.p\right|_{[\varepsilon, 1]}}$ for some $\varepsilon \in(0,1)$. Thus $d\left(x_{0}^{\prime}, y_{0}\right)<d\left(x_{0}, y_{0}\right)=L_{\gamma_{2}}+L_{p}$, a contradiction. Therefore ind $_{+}\left(x_{0}, y_{0}\right)=0$ and so $\left(x_{0}, y_{0}\right)$ is an index zero configuration. If case (b) applies and if $\operatorname{ind}_{+}\left(x, y_{0}\right)>0$, push $x$ away from $y_{0}$ as above to obtain a point $x_{0}$ with $\operatorname{ind}_{+}\left(x_{0}, y_{0}\right)=\operatorname{ind}_{+}\left(y_{0}, x_{0}\right)=0$. This defines a continuous path in $F_{r}(\Gamma, 2)$ from $(x, y)$ to an index zero configuration $\left(x_{0}, y_{0}\right)$. Hence, $P$ contains at least one configuration $\left(x_{0}, y_{0}\right) \in Z_{r}$, and so contains at least one path component of $Z_{r}$.

Example 2.5 Let $T$ be the labelled tree shown in Figure 4. The index zero config-

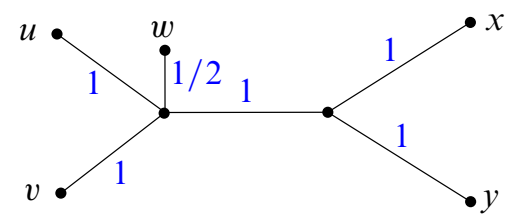

Figure 4: The labelled tree $T$

urations are $(x, y),(x, u),(x, v),(x, w),(y, w),(y, u),(y, v),(w, u),(w, v)$ and $(u, v)$, together with their images under the involution $\left(x_{1}, x_{2}\right) \mapsto\left(x_{2}, x_{1}\right)$. Applying Proposition 2.4 gives the following table.

\begin{tabular}{c|c} 
interval & upper bound on $b_{0}\left(F_{r}(T, 2)\right)$ \\
\hline $0<r \leq 3 / 4$ & 20 \\
$3 / 4<r \leq 1$ & 16 \\
$1<r \leq 5 / 4$ & 12 \\
$5 / 4<r \leq 3 / 2$ & 8
\end{tabular}

\subsection{The graph $G_{r}$}

We now introduce a graph $G_{r}=G_{r}(\Gamma)$ such that there is a bijection between the path components of $G_{r}$ and the path components of $F_{r}(\Gamma, 2)$. We view $G_{r}$ as a one-dimensional $\mathrm{CW}$ complex, so that $G_{r}$ is permitted to contain loops and multiple edges.

Definition 2.6 We write $\operatorname{Crit}(r)$ for the set of all critical configurations $(x, y)$ such that $d(x, y) \geq 2 r$. 
The construction of $G_{r}$ is as follows.

(1) Find $\operatorname{Crit}(r)$. For each isolated configuration $(x, y) \in \operatorname{Crit}(r)$, construct a corresponding vertex $(x, y) \in G_{r}$.

(2) For each cycle $C$ which contains nonisolated configurations in $\operatorname{Crit}(r)$ (namely, antipodal points on $C$ ) construct a copy of $C=S^{1}$ in $G_{r}$, realised as the pairs $(x, y)$ of antipodal points on $C$. If there are common pairs of antipodal points between cycles, then identify these in the copies of the cycles in $G_{r}$.

(3) To construct the remaining edges of $G_{r}$, let $(x, y) \in \operatorname{Crit}(r)$ be an isolated configuration which does not have index zero. There is a path in $F_{r}(\Gamma, 2)$ from $(x, y)$ to an index zero configuration, which need not be unique; see Proposition 2.4. For each point $V=(x, s)$ or $V=(t, y)$ of $G_{r}$ which is joined to $(x, y)$ via such a path, construct edges in $G_{r}$ from $(x, y)$ to $V$, one for each distinct path and making the appropriate identifications if the paths overlap. If $V$ is not index zero, repeat this procedure for $V$.

(4) Apply step (3) to any pair $(x, y)$ not of index zero lying on a cycle $C$ from step (2). If $(x, y) \in C$ is not critical, it may be necessary to move both $x$ and $y$ to reach a configuration $V$ in $G_{r}$. In this case, insert edges in $G_{r}$ from $(x, y)$ to $V$ as in step (3). Also apply step (3), if no corresponding edge has already been constructed, to any isolated index zero configuration joined by a path in $F_{r}(\Gamma, 2)$ to elements of $G_{r}$ from steps (1) and (2).

(5) If any circle from step (2) is left isolated after these steps, then designate one of its points to be a vertex of $G_{r}$. This is simply to ensure that $G_{r}$ is a graph.

Remark 2.7 We emphasise that $G_{r}$ is dependent on $r$. If $r, s \in\left(0, \frac{1}{2} \operatorname{diam}(\Gamma)\right]$ and $r<s$, then $G_{s}$ is a subgraph of $G_{r}$, possibly equal to $G_{r}$.

Example 2.8 Let $\Gamma$ be the $Y$-graph as shown.

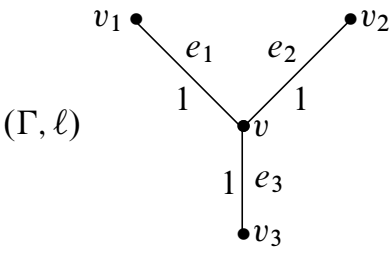

For $0<r \leq 1 / 2$, $\operatorname{Crit}(r)$ comprises the 12 pairs of distinct vertices of $\Gamma$, so $G_{r}$ has 12 vertices; see Figure 5. For each $i \in\{1,2,3\}, v$ can be pushed away from $v_{i}$ in two distinct directions, so $G_{r}$ has 12 edges. If $1 / 2<r \leq 1$, then $G_{r}$ comprises the six vertices $\left(v_{i}, v_{j}\right), i \neq j$, from Figure 5 . 


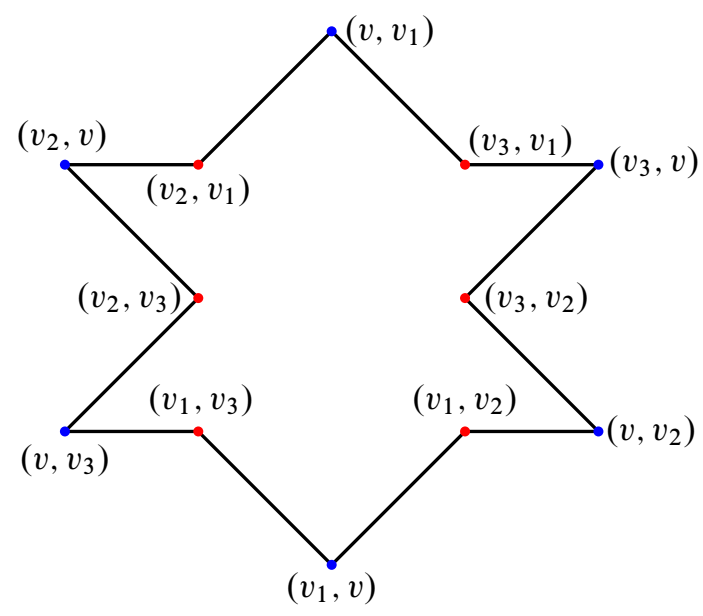

Figure 5: $G_{r}$ for $0<r \leq 1 / 2$

Example 2.9 Let $\Gamma=S^{1} \vee S^{1}$ have the graph structure in Figure 6. For $0<r \leq 1 / 4$, the graph $G_{r}$ is shown on the left of Figure 7. For $1 / 4<r \leq 1 / 2, G_{r}$ is shown on the right of Figure 7, and for $1 / 2<r \leq 3 / 4, G_{r}$ comprises the two points $(x, y)$ and $(y, x)$.

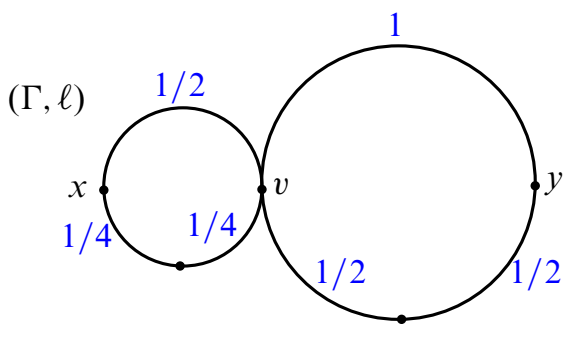

Figure 6: The graph structure on $\Gamma=S^{1} \vee S^{1}$

We now make the following observation.

Lemma 2.10 There is an embedding $G_{r} \hookrightarrow F_{r}(\Gamma, 2)$.

Proof From the construction of $G_{r}$, the isolated configurations from step (2) and the circles from step (3) are included in $F_{r}(\Gamma, 2)$. Suppose that $E$ is an edge from step (4) or step (5), joining two configurations $(x, y)$ and $\left(x^{\prime}, y^{\prime}\right)$. Let $\gamma:[0,1] \rightarrow F_{r}(\Gamma, 2)$ be the constant-speed path from $(x, y)$ to $\left(x^{\prime}, y^{\prime}\right)$ corresponding to $E$. Identifying $\left(E, \partial_{0} E\right)$ with $([0,1], 0)$ gives an embedding $E \rightarrow F_{r}(\Gamma, 2)$. Patching together these embeddings over all edges of $G_{r}$ gives an embedding $G_{r} \hookrightarrow F_{r}(\Gamma, 2)$. 


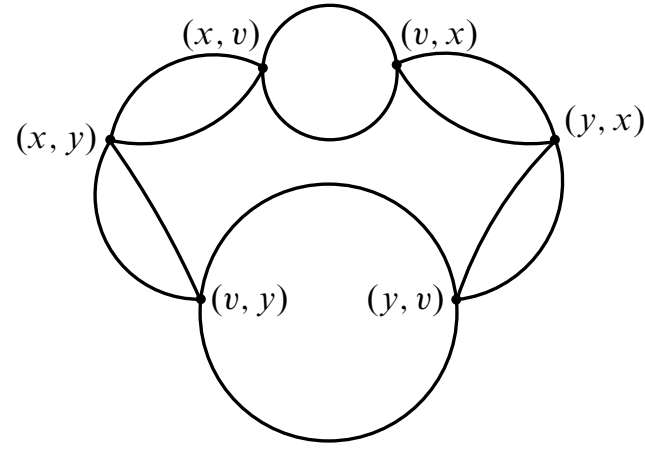

$G_{r}$ for $0<r \leq 1 / 4$

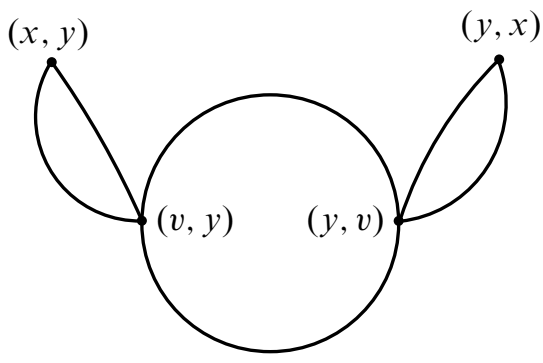

$G_{r}$ for $0<r \leq 1 / 2$

Figure 7: The graphs $G_{r}$

The following statement is the main result of this section.

Theorem 2.11 We have $b_{0}\left(F_{r}(\Gamma, 2)\right)=b_{0}\left(G_{r}\right)$.

Proof We show that the inclusion map $\iota$ : $G_{r} \hookrightarrow F_{r}(\Gamma, 2)$ induces a bijection

$$
\iota_{*}: \pi_{0}\left(G_{r}\right) \rightarrow \pi_{0}\left(F_{r}(\Gamma, 2)\right) .
$$

By Proposition 2.4, each path component of $F_{r}(\Gamma, 2)$ contains an index zero configuration $\left(x_{0}, y_{0}\right)$ with $d\left(x_{0}, y_{0}\right) \geq 2 r$. Since $\left(x_{0}, y_{0}\right) \in G_{r}, \iota_{*}$ is surjective. Now suppose that $\iota_{*}([x, y])=\iota_{*}\left(\left[x^{\prime}, y^{\prime}\right]\right)$, so that there is a path in $F_{r}(\Gamma, 2)$ from $(x, y)$ to $\left(x^{\prime}, y^{\prime}\right)$. By Proposition 2.4, it may be assumed that $(x, y)$ and $\left(x^{\prime}, y^{\prime}\right)$ are index zero configurations. By the construction of $G_{r}$, any pair of index zero configurations joined by a path in $F_{r}(\Gamma, 2)$ are joined by a path in $G_{r}$, so $[(x, y)]=\left[\left(x^{\prime}, y^{\prime}\right)\right]$ in $G_{r}$. Hence, $\iota_{*}$ is injective.

Corollary 2.12 An algorithm for computing $b_{0}\left(F_{r}(\Gamma, 2)\right)$ given $\Gamma$ and $r$ is as follows. We construct the graph $G_{r}$ and compute the Betti number $b_{0}\left(G_{r}\right)$.

Example 2.13 Let $T$ be the labelled tree from Example 2.5. Constructing the graphs $\left\{G_{r}\right\}_{r>0}$ and applying Theorem 2.11 gives the following table.

\begin{tabular}{c|c} 
interval & $b_{0}\left(F_{r}(T, 2)\right)$ \\
\hline $0<r \leq 1 / 2$ & 1 \\
$1 / 2<r \leq 3 / 4$ & 10 \\
$3 / 4<r \leq 1$ & 6 \\
$1<r \leq 5 / 4$ & 12 \\
$5 / 4<r \leq 3 / 2$ & 8
\end{tabular}




\section{PL Morse-Bott theory}

In this section we describe our main tool for studying the homotopy type of the configuration spaces $\left\{F_{r}(\Gamma, 2)\right\}_{r>0}$. PL Morse theory was developed by Bestvina and Brady [6] (see also Bestvina [5]) and we extend their results to the Morse-Bott case. The difference is that we allow our functions to be constant on cells of positive dimension.

\subsection{Definitions}

Definition 3.1 (See [5, Definition 2.1].) An affine polytope complex of dimension $m$ is a CW complex $X$ in which each closed cell $F$ is equipped with a characteristic function $\chi_{F}: C_{F} \rightarrow X$ such that

(i) $C_{F}$ is a convex polyhedral cell in $\mathbb{R}^{m}$ for each $F$;

(ii) $\chi_{F}$ is an embedding for each closed cell $F$;

(iii) the restriction of $\chi_{F}$ to any face of $C_{F}$ coincides with the characteristic function of another cell precomposed with an affine homeomorphism of $\mathbb{R}^{m}$.

Let $X$ be an affine polytope complex of dimension $m$.

Definition 3.2 A continuous map $f: X \rightarrow \mathbb{R}$ is an affine Morse-Bott function if $f \circ \chi_{F}: C_{F} \rightarrow \mathbb{R}$ extends to an affine function $\mathbb{R}^{m} \rightarrow \mathbb{R}$ for each cell $F \subset X$.

Remark 3.3 We emphasise that $f \circ \chi_{F}$ may be constant on a cell $F \subset X$ with $\operatorname{dim} F>0$.

Let $f: X \rightarrow \mathbb{R}$ be an affine Morse-Bott function.

Definition 3.4 A cell $F$ of $X$ is critical if $f$ is constant on $F$.

Remark 3.5 In particular, all 0 -cells of $X$ are critical.

Definition 3.6 A number $R \in \mathbb{R}$ is a critical level of $f$ if there exists a cell $F$ of $X$ on which $f$ is constant with value $R$.

Definition 3.7 The union of all critical cells is the critical subcomplex of $f$, and is denoted by $C(f)$.

Remarks 3.8 (i) If $f$ is constant on an open cell $e \subset X$, then by continuity $f$ is constant on $\bar{e}$. Hence, $C(f)$ is indeed a subcomplex of $X$. 
(ii) $\quad C(f)=\bigsqcup_{R} C(f, R)$ is the disjoint union of the subcomplexes $C(f, R)$, where $R$ ranges over all critical levels of $f$ and $C(f, R)$ is the union of all critical cells $K$ such that $\left.f\right|_{K} \equiv R$.

(iii) Writing $C(f)=\bigsqcup_{\alpha \in A} Z_{\alpha}$ as the union of its connected components refines the decomposition from (ii), that is, for each $\alpha \in A$ there is a critical level $R$ such that $Z_{\alpha} \subset C(f, R)$.

Definition 3.9 A noncritical cell $K$ of $X$ is descending if $\left.f\right|_{K}$ achieves its maximum on a subcomplex of $K \cap C(f)$.

Notation 3.10 If $f: X \rightarrow \mathbb{R}$ is an affine Morse-Bott function and $c \in \mathbb{R}$, we write $X^{c}:=f^{-1}((-\infty, c])$.

\subsection{Descending sets and links}

We now define the descending set $D_{\downarrow}\left(Z_{\alpha}\right)$ and the descending link $\operatorname{Lk}_{\downarrow}\left(Z_{\alpha}\right)$ of a connected component $Z_{\alpha}$ of the critical subcomplex $C(f)$. Suppose that $\left.f\right|_{Z_{\alpha}} \equiv R$. Choose $S<R$ such that the interval $[S, R)$ does not contain any critical level of $f$.

Definition 3.11 The descending set $D_{\downarrow}\left(Z_{\alpha}\right)$ of $Z_{\alpha}$ is

$$
D_{\downarrow}\left(Z_{\alpha}\right):=f^{-1}([S, R]) \cap \bigcup K,
$$

where the union is taken over the cells $K$ of $X$ such that $\left.f\right|_{K}$ achieves its maximum on $K \cap Z_{\alpha}$.

Remark 3.12 $D_{\downarrow}\left(Z_{\alpha}\right)$ is a closed subspace of $X$ containing $Z_{\alpha}$.

Definition 3.13 The descending link $\operatorname{Lk}_{\downarrow}\left(Z_{\alpha}\right)$ of $Z_{\alpha}$ is

$$
\operatorname{Lk}_{\downarrow}\left(Z_{\alpha}\right):=f^{-1}(\{S\}) \cap \bigcup K=f^{-1}(\{S\}) \cap D_{\downarrow}\left(Z_{\alpha}\right) .
$$

Remark 3.14 Definition 3.11 and Definition 3.13 are independent of the choice of $S$ (provided $[S, R$ ) contains no critical level of $f$ ), because each cell of $X$ is PL homeomorphic to a convex polyhedron in $\mathbb{R}^{m}$.

Example 3.15 Consider the hexagon $X$ shown in Figure 8, comprising seven 0-cells, eleven 1-cells and five 2-cells. Let $f: X \rightarrow[0,1]$ be the height function as shown. The subcomplex $C(f, R)$ has two connected components $Z_{1}=F$ and $Z_{2}=\{w\}$. The descending set $D_{\downarrow}\left(Z_{1}\right)$ and the descending link $\operatorname{Lk}_{\downarrow}\left(Z_{1}\right)$ are the subsets of $K \cup L$ shown in Figure 9. On the other hand, $D_{\downarrow}\left(Z_{2}\right) \subset E$ is an interval, and $\operatorname{Lk}_{\downarrow}\left(Z_{2}\right)$ is a single point. 


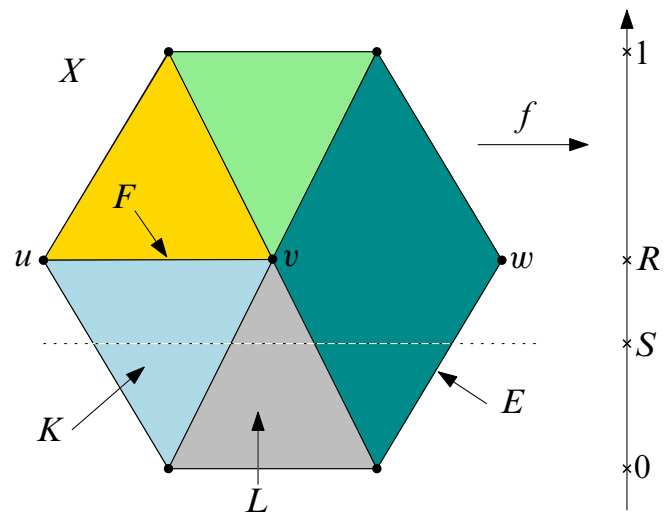

Figure 8: The hexagon $X$

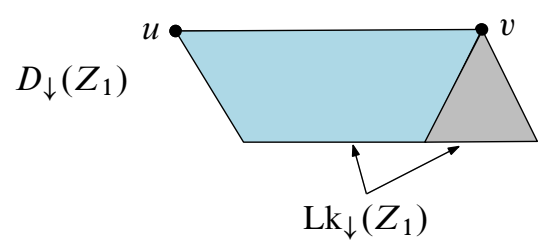

Figure 9

Lemma 3.16 There is a deformation retraction of $D_{\downarrow}\left(Z_{\alpha}\right)$ onto $Z_{\alpha}$.

Proof The proof is very similar to [5, Proposition 2.4]. For $j \in\{0, \ldots, m\}$, let

$$
A_{j}:=Z_{\alpha} \cup\left(D_{\downarrow}\left(Z_{\alpha}\right) \cap X^{(j)}\right),
$$

where $X^{(j)}$ is the $j$-skeleton of $X$. In particular, $A_{0}=Z_{\alpha}$ and $A_{m}=D_{\downarrow}\left(Z_{\alpha}\right)$. It suffices to show that $A_{j}$ deformation retracts onto $A_{j-1}$ for each $j \in\{1, \ldots, m\}$. Let $L$ be a descending $j$-cell. Identify $L$ with the convex $j$-cell $C_{L}$ in $\mathbb{R}^{m}$ via the characteristic map $\chi_{L}: C_{L} \rightarrow L$. By possibly precomposing with a partial affine homeomorphism of $\mathbb{R}^{m}$, it may be assumed that $f$ is a height function on $L$ (since $f$ is not constant on $L)$. Then $f^{-1}([S, R]) \cap L$ may be identified with a convex polytope $K$ in $\mathbb{R}^{m}$, and $f^{-1}(\{S\}) \cap L$ is the lower face of $K$. The set $f^{-1}(\{R\}) \cap L$ is a convex polyhedron of dimension strictly smaller than $\operatorname{dim} K$. The polytope $K$ has the following property: there is a deformation retraction of $K$ onto its boundary $\partial K$ minus the lower $(j-1)$-dimensional face. Combining these deformation retractions over all such $j$-cells $L$ of $X$ gives a deformation retraction of $A_{j}$ onto $A_{j-1}$. 
Example 3.17 Consider the hexagon from Figure 8. The steps of the deformation retraction of $D_{\downarrow}\left(Z_{1}\right)$ onto $Z_{1}$ from Lemma 3.16 are shown as follows.

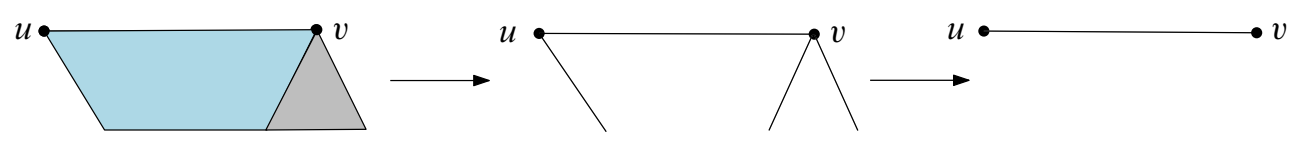

Cell structure The descending link $\operatorname{Lk}_{\downarrow}\left(Z_{\alpha}\right)$ of a connected component $Z_{\alpha}$ of $C(f)$ may be equipped with a cell structure as follows. For each $j \in\{0,1, \ldots, m-1\}$, the $j$-cells of $\operatorname{Lk}_{\downarrow}\left(Z_{\alpha}\right)$ are the connected components of the intersection

$$
\operatorname{Lk}_{\downarrow}\left(Z_{\alpha}\right) \cap \bigcup\left(K^{(j+1)} \backslash K^{(j)}\right),
$$

where $K^{(\ell)}$ is the $\ell$-skeleton of $K$. Here, the union is over the cells $K$ of $X$ such that $\left.f\right|_{K}$ achieves its maximum on $K \cap Z_{\alpha}$.

There is also a surjection

$$
h_{\alpha}: \operatorname{Lk}_{\downarrow}\left(Z_{\alpha}\right) \rightarrow Z_{\alpha}
$$

defined inductively on the skeleta of $\operatorname{Lk}_{\downarrow}\left(Z_{\alpha}\right)$ as follows.

(i) Let $v$ be a vertex of $\operatorname{Lk}_{\downarrow}\left(Z_{\alpha}\right)$. There exists a unique 1-cell of $X$ connecting $v$ to a vertex $v^{\prime}$ in $Z_{\alpha}$ (this 1 -cell is unique because there are no critical levels of $f$ in the interval $[S, R))$. Define $h_{\alpha}(v)=v^{\prime}$.

(ii) The map $h_{\alpha}$ is cellular, and on each $j$-cell of $\operatorname{Lk}_{\downarrow}\left(Z_{\alpha}\right), h_{\alpha}$ is either constant or maps linearly onto a cell of $Z_{\alpha}$ of dimension at most $j$.

In general, the map $h_{\alpha}: \operatorname{Lk}_{\downarrow}\left(Z_{\alpha}\right) \rightarrow Z_{\alpha}$ satisfies the following property. The descending set $D_{\downarrow}\left(Z_{\alpha}\right)$ is a copy of the mapping cylinder $M\left(h_{\alpha}\right)$, with base $f^{-1}(\{S\}) \cap$ $D_{\downarrow}\left(Z_{\alpha}\right)=\operatorname{Lk}_{\downarrow}\left(Z_{\alpha}\right)$ and top $f^{-1}(\{R\}) \cap D_{\downarrow}\left(Z_{\alpha}\right)=Z_{\alpha}$.

Example 3.18 Let $T$ be the solid tetrahedron in Figure 10. It is an affine polytope complex with four $0-$ cells, six 1-cells, four 2-cells and one 3-cell $K$. Let $f: T \rightarrow \mathbb{R}$ be the affine Morse-Bott height function as shown. The critical subcomplex $C(f)$ has three components, one of which is $Z_{1}=C(f, R)=F$. The descending link $\operatorname{Lk}_{\downarrow}\left(Z_{1}\right)$ is a 2-dimensional convex polyhedron with four vertices $v_{1}, \ldots, v_{4}$, four 1 -cells $e_{1}, \ldots, e_{4}$ and one $2-$ cell. The map $h_{1}: \operatorname{Lk}_{\downarrow}\left(Z_{1}\right) \rightarrow Z_{1}$ is specified as follows: $h_{1}$ maps $\bar{e}_{1}$ to $v$ and $\bar{e}_{2}$ to $w$, whereas $\bar{e}_{3}, \bar{e}_{4}$ and every segment parallel to $\bar{e}_{3}, \bar{e}_{4}$ is mapped linearly onto $F$. 


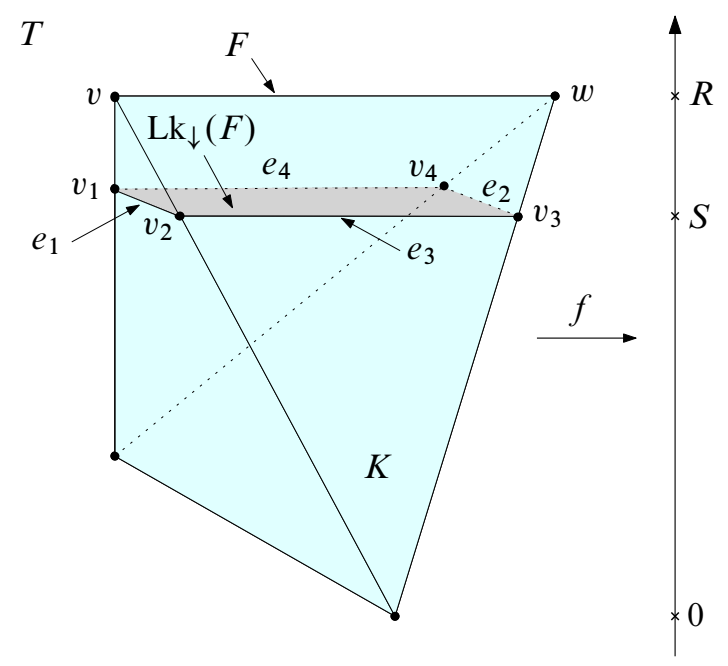

Figure 10: The solid tetrahedron $T$

\subsection{Morse-Bott theory for affine polytope complexes}

Let $f: X \rightarrow \mathbb{R}$ be an affine Morse-Bott function. We now study the homotopy type of the sets $\left\{X^{c}\right\}_{c \in \mathbb{R}}$. First, we consider the case when there are no critical levels in an interval.

Proposition 3.19 Let $R<R^{\prime}$ be such that there are no critical levels of $f: X \rightarrow \mathbb{R}$ in the interval $\left(R, R^{\prime}\right]$. Then $X^{R^{\prime}}$ deformation retracts onto $X^{R}$.

Proof For $j \in\{0,1, \ldots, m\}$, let

$$
A_{j}=X^{R} \cup\left(X^{R^{\prime}} \cap X^{(j)}\right),
$$

so that $A_{0}=X^{R}$ and $A_{m}=X^{R^{\prime}}$. It suffices to show that $A_{j}$ deformation retracts onto $A_{j-1}$ for each $j \in\{1, \ldots, m\}$. Consider a $j$-cell $L$ of $X$ such that $L \cap$ $f^{-1}\left(\left(R, R^{\prime}\right]\right) \neq \varnothing$. Identify $L$ with the convex $j$-cell $C_{L}$ in $\mathbb{R}^{m}$ via the characteristic map $\chi_{L}: C_{L} \rightarrow L$. By possibly precomposing with a partial affine homeomorphism of $\mathbb{R}^{m}$, it may be assumed that $f$ is a height function on $L$ (since $f$ is not constant on $L)$. Then $f^{-1}\left(\left[R, R^{\prime}\right]\right) \cap L$ may be identified with a convex polytope $K$ in $\mathbb{R}^{m}$. The set $f^{-1}\left(\left\{R^{\prime}\right\}\right) \cap L$ is the top face of the polytope $K$. The set $f^{-1}(\{R\}) \cap L$ is a convex polyhedron of dimension strictly smaller than $\operatorname{dim} K$. The polytope $K$ has the following property: there is a deformation retraction of $K$ onto its boundary $\partial K$ minus the top $(j-1)$-dimensional face. Combining these deformation retractions over all $j$-cells $L$ of $X$ with $L \cap f^{-1}\left(\left(R, R^{\prime}\right]\right) \neq \varnothing$ gives a deformation retraction of $A_{j}$ onto $A_{j-1}$. 
Example 3.20 Consider the solid pyramid $X$ in Figure 11. It is an affine polytope complex with five 0 -cells, eight 1 -cells, five 2 -cells and one 3 -cell. Let $f: X \rightarrow \mathbb{R}$
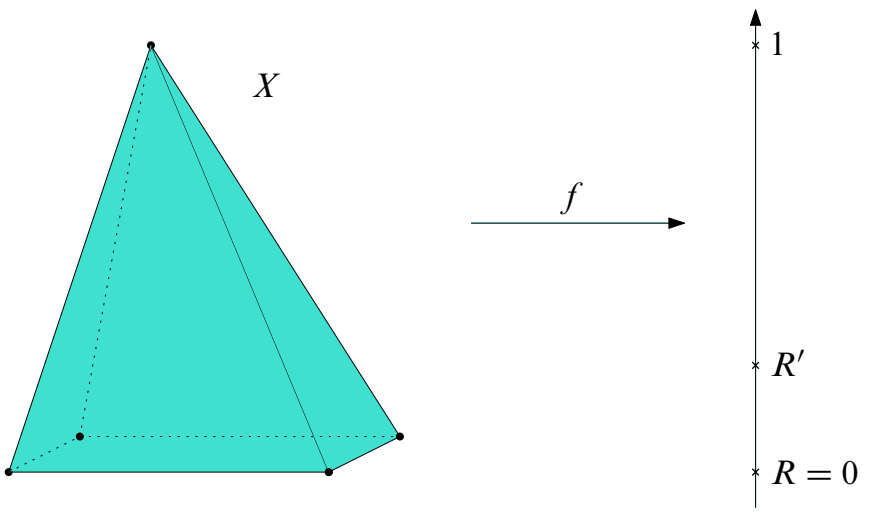

Figure 11: The pyramid $X$ and the affine Morse-Bott function $f$

be the affine Morse-Bott function as shown. Here $R^{\prime} \in(0,1)$, and the base of $X$ is at level $R=0$. In this case, $\operatorname{dim} X=3$ and the steps of the deformation retraction from Proposition 3.19 are shown in Figure 12.

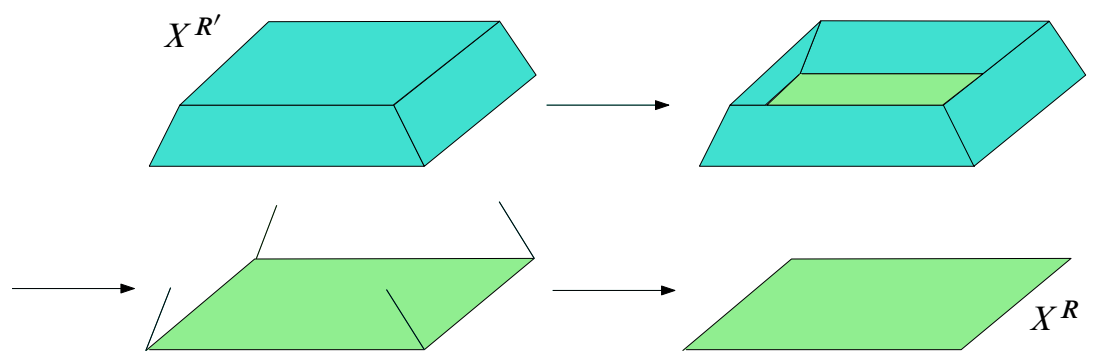

Figure 12: The steps of the deformation retraction from Proposition 3.19

We now consider the case when there is exactly one critical level in an interval. Let $a<R<b$ be such that $R$ is the only critical level of $f$ in $[a, b]$, and write $C(f, R)=$ $\bigsqcup_{i=1}^{n} Z_{i}$ as the disjoint union of its connected components.

Proposition 3.21 $\left(X^{b}, X^{a}\right)$ is homotopy equivalent to the pair $\left(Q, X^{a}\right)$, where $Q \subset X^{R}$ is the space obtained from $X^{a}$ by attaching the mapping cylinders $\left\{M\left(h_{i}\right)=D_{\downarrow}\left(Z_{i}\right)\right\}_{i=1}^{n}$ as follows: for each $i \in\{1, \ldots, n\}$, identify the copies of $\operatorname{Lk}_{\downarrow}\left(Z_{i}\right)$ contained in $M\left(h_{i}\right)$ and $X^{a}$. 
Proof In view of Proposition 3.19, $X^{b}$ deformation retracts onto $X^{R}$. It suffices to show that $f^{-1}([a, R])$ deformation retracts onto its subspace $Q \cap f^{-1}([a, R])$. For $j \in\{0,1, \ldots, m\}$, let

$$
A_{j}=\left(Q \cup X^{(j)}\right) \cap f^{-1}([a, R]),
$$

so that $A_{0}=Q \cap f^{-1}([a, R])$ and $A_{m}=f^{-1}([a, R])$. Again, we show that $A_{j}$ deformation retracts onto $A_{j-1}$ for each $j \in\{1, \ldots, m\}$. Let $L$ be a $j$-cell of $X$ such that $f^{-1}([a, R]) \cap L \neq \varnothing$ and $L$ does not contain a $(j-1)$-cell of $C(f, R)$ in its boundary. Identify $L$ with the convex polyhedral cell $C_{L}$ in $\mathbb{R}^{m}$; it may be assumed that $f$ is a height function on $L$. Then $f^{-1}([a, R]) \cap L$ is a $j$-dimensional convex polytope in $\mathbb{R}^{m}$, with top face $f^{-1}(\{R\}) \cap L$ not a cell of $C(f, R)$. There is a deformation retraction of this polytope onto its boundary minus the top face. Hence, $A_{j}$ deformation retracts onto $A_{j-1}$.

Example 3.22 Consider the Morse-Bott function $f: X \rightarrow[0,1]$ from Figure 8. Let $a<R<b$ be as shown in Figure 13. The subcomplex $C(f, R)$ has two connected components $Z_{1}=F$ and $Z_{2}=\{w\}$. The mapping cylinders $M\left(h_{1}\right)$ and $M\left(h_{2}\right)$ coincide with $D_{\downarrow}\left(Z_{1}\right)$ and $D_{\downarrow}\left(Z_{2}\right)$, respectively. The space $X^{b}$ deformation retracts onto $X^{R}$, and the pair $\left(X^{R}, X^{a}\right)$ deformation retracts onto $\left(Q, X^{a}\right)$ as in Figure 14.

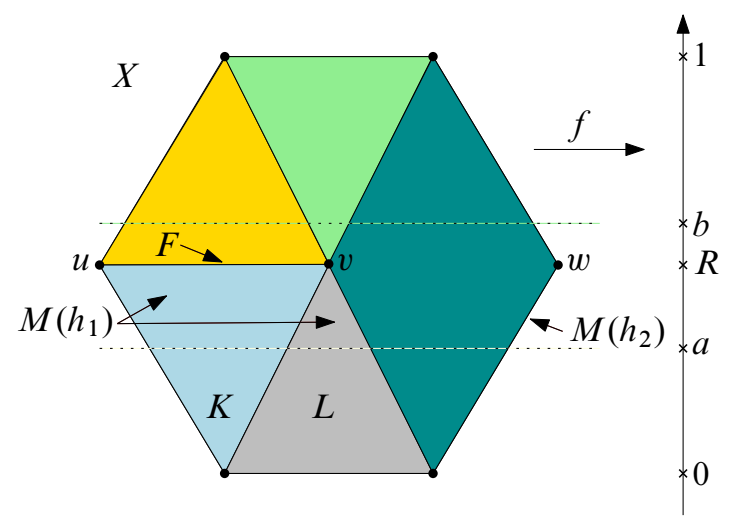

Figure 13: The mapping cylinders $M\left(h_{1}\right)$ and $M\left(h_{2}\right)$

Remark 3.23 There are analogues of Propositions 3.19 and 3.21 for the family $\left\{X_{c}\right\}_{c \in \mathbb{R}}$, where $X_{c}:=f^{-1}([c, \infty))$ for each $c \in \mathbb{R}$. In fact, if $f: X \rightarrow \mathbb{R}$ is an affine Morse-Bott function on $X$, then so is $-f: X \rightarrow \mathbb{R}$. 

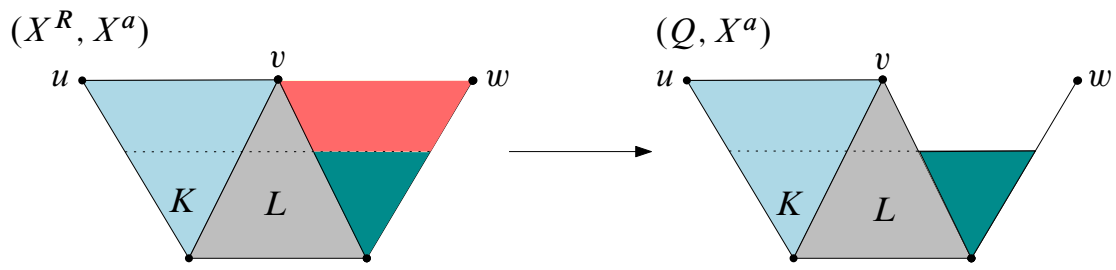

Figure 14: Deformation retraction of $\left(X^{R}, X^{a}\right)$ onto $\left(Q, X^{a}\right)$

Corollary 3.24 Assume that $C(f, R)=Z$ is connected, and let $h: \operatorname{Lk}_{\downarrow}(Z) \rightarrow Z$ be the map defined in Section 3.2 (in particular, $M(h)=D_{\downarrow}(Z)$ ). Then the inclusion $\left(M(h), \mathrm{Lk}_{\downarrow}(Z)\right) \hookrightarrow\left(X^{b}, X^{a}\right)$ induces homology isomorphisms.

Proof The map $\left(Q, X^{a}\right) \hookrightarrow\left(X^{b}, X^{a}\right)$ is a homotopy equivalence by Proposition 3.21. By excision, the inclusion $\left(M(h), \operatorname{Lk}_{\downarrow}(Z)\right) \hookrightarrow\left(Q, X^{a}\right)$ induces isomorphisms on homology.

Corollary 3.25 Under the assumptions of Corollary 3.24,

$\operatorname{rk} H_{j}\left(X^{b}, X^{a}\right)=\operatorname{rkcoker}\left(\iota_{*}: H_{j}\left(\operatorname{Lk}_{\downarrow}(Z)\right) \rightarrow H_{j}\left(D_{\downarrow}(Z)\right)\right)$

$$
+\operatorname{rk} \operatorname{ker}\left(\iota_{*}: H_{j-1}\left(\operatorname{Lk}_{\downarrow}(Z)\right) \rightarrow H_{j-1}\left(D_{\downarrow}(Z)\right)\right)
$$

for each $j \geq 0$.

Proof By replacing $H_{j}\left(X^{b}, X^{a}\right)$ with $H_{j}\left(D_{\downarrow}(Z), \mathrm{Lk}_{\downarrow}(Z)\right)$, the long exact sequence of the pair $\left(D_{\downarrow}(Z), \mathrm{Lk}_{\downarrow}(Z)\right)=\left(M(h), \mathrm{Lk}_{\downarrow}(Z)\right)$ becomes:

$$
\begin{aligned}
\cdots \rightarrow H_{j}\left(\operatorname{Lk}_{\downarrow}(Z)\right) \stackrel{\iota_{*}}{\rightarrow} H_{j}\left(D_{\downarrow}(Z)\right) & \rightarrow H_{j}\left(X^{b}, X^{a}\right) \\
& \rightarrow H_{j-1}\left(\operatorname{Lk}_{\downarrow}(Z)\right) \stackrel{\iota_{*}}{\rightarrow} H_{j-1}\left(D_{\downarrow}(Z)\right) \rightarrow \cdots
\end{aligned}
$$

Remark 3.26 Now suppose that $C(f, R)=\coprod_{i=1}^{n} Z_{i}$ has $n$ connected components $Z_{1}, \ldots, Z_{n}$. The analogues of Corollaries 3.24 and 3.25 are as follows.

(i) The inclusion $\coprod_{i=1}^{n}\left(M\left(h_{i}\right), \mathrm{Lk}_{\downarrow}\left(Z_{i}\right)\right) \hookrightarrow\left(X^{b}, X^{a}\right)$ induces isomorphisms on homology.

(ii) For each $j \geq 0$,

$$
\begin{aligned}
\operatorname{rk} H_{j}\left(X^{b}, X^{a}\right)=\sum_{i=1}^{n}(\operatorname{rk} & \operatorname{coker}\left(\iota_{*}: H_{j}\left(\operatorname{Lk}_{\downarrow}\left(Z_{i}\right)\right) \rightarrow H_{j}\left(D_{\downarrow}\left(Z_{i}\right)\right)\right. \\
& \left.+\operatorname{rkker}\left(\iota_{*}: H_{j-1}\left(\operatorname{Lk}_{\downarrow}\left(Z_{i}\right)\right) \rightarrow H_{j-1}\left(D_{\downarrow}\left(Z_{i}\right)\right)\right)\right) .
\end{aligned}
$$




\section{The metric is an affine Morse-Bott function}

Recall that the distance $d(x, y)$ between points $x, y \in \Gamma$ is the length of the shortest arc connecting $x$ and $y$. In this section we verify that $d$ is an affine Morse-Bott function on $\Gamma \times \Gamma$. This enables us to apply the general statements from Section 3 and obtain information on the homotopy type of the spaces $\left\{F_{r}(\Gamma, 2)\right\}_{r>0}$. With respect to some $\mathrm{CW}$ decomposition of $\Gamma \times \Gamma$, we need to show that

(i) $\Gamma \times \Gamma$ is an affine polytope complex (APC);

(ii) for each closed cell $F$ of $\Gamma \times \Gamma$, the composition

$$
d \circ \chi_{F}: C_{F} \rightarrow \mathbb{R}
$$

extends to an affine map $\mathbb{R}^{2} \rightarrow \mathbb{R}$. Here, $\chi_{F}: C_{F} \rightarrow F$ is a characteristic function of $F$ (see Definition 3.1), and $C_{F}$ is a convex polyhedral cell in $\mathbb{R}^{2}$ of dimension $\operatorname{dim} F$.

We first define a condition (the cycle inequality), similar to the triangle inequality, which holds in $\Gamma$ under sufficient subdivision.

The cycle inequality A cycle in $\Gamma$ is a subgraph homeomorphic to $S^{1}$. Since $\Gamma$ is simple, each cycle is the union of at least three closed edges. If $C$ is a cycle, then we write $C=\left(e_{1}, \ldots, e_{K}\right)$ to indicate that $C=\bigcup_{i=1}^{K} \bar{e}_{i}$ and edge $e_{j}$ is incident to edges $e_{j+1}$ and $e_{j-1}$, taking subscripts modulo $K$; see Figure 15 . A cycle $C=\left(e_{1}, \ldots, e_{K}\right)$

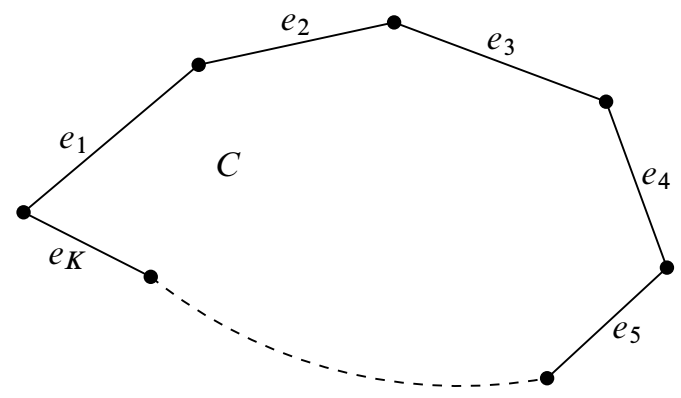

Figure 15: A cycle $C=\left(e_{1}, \ldots, e_{K}\right)$ in a simple graph

satisfies the cycle inequality if $\ell\left(e_{i}\right) \leq \sum_{1 \leq j \leq K, j \neq i} \ell\left(e_{j}\right)$ for each $i \in\{1, \ldots, K\}$. An edge-labelled graph $\Gamma$ is said to satisfy the cycle inequality if each of its cycles satisfies the cycle inequality. It is easy to check that any simple edge-labelled graph admits a subdivision satisfying the cycle inequality.

We now assume that $\Gamma$ satisfies the cycle inequality (otherwise, replace $\Gamma$ with a suitable subdivision). In the remainder of this section we verify the following statement. 
Proposition 4.1 The product $C W$ structure on $\Gamma \times \Gamma$ admits a subdivision such that (i) and (ii) hold.

Proof Since $\Gamma$ is simple, it is a cubical complex of dimension one. Hence, the product $\Gamma \times \Gamma$ is a cubical complex of dimension two. Any finite-dimensional cubical complex is an $\mathrm{APC},{ }^{1}$ so $\Gamma \times \Gamma$ is an APC with respect to the product $\mathrm{CW}$ structure. So (i) holds.

We now show that $\Gamma \times \Gamma$ admits a subdivision such that (ii) holds. Choose an identification $\phi_{e}: \bar{e} \rightarrow[0,1]$ of each closed edge $\bar{e}$ with $[0,1]$, and write $\partial_{i} e=\phi_{e}^{-1}(i)$ for $i \in\{0,1\}$. A closed $2-$ cell $F$ of $\Gamma \times \Gamma$ has the form $F=\bar{e} \times \bar{f}$, where $e$ and $f$ are edges. Set $C_{F}=[0,1] \times[0,1]$ and $\chi_{F}(i, j)=\left(\partial_{i} e, \partial_{j} f\right)$ for all $i, j \in\{0,1\}$. If $e=f$, then since the cycle inequality is satisfied, $\left.d\right|_{F}$ has the form

$$
d(x, y)=\ell(e)\left|\phi_{e}(x)-\phi_{e}(y)\right|, \quad(x, y) \in F,
$$

where $\ell(e)$ is the label of $e$. In this case, add a diagonal $1-$ cell to $F=\bar{e} \times \bar{e}$ as shown in Figure 16, creating two triangular 2-cells $F_{1}$ and $F_{2}$. If $\chi_{F_{j}}: C_{F_{j}} \rightarrow F_{j}$ is a characteristic function of $F_{j}$ for $j \in\{1,2\}$, then $d \circ \chi_{F_{j}}$ extends to an affine map $\mathbb{R}^{2} \rightarrow \mathbb{R}$, for $j \in\{1,2\}$; see Figure 17. Now assume that $e$ and $f$ are distinct. There

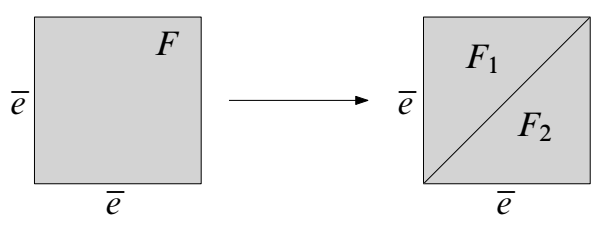

Figure 16: Subdividing a diagonal 2-cell $F=\bar{e} \times \bar{e}$

$$
2|x-y|
$$

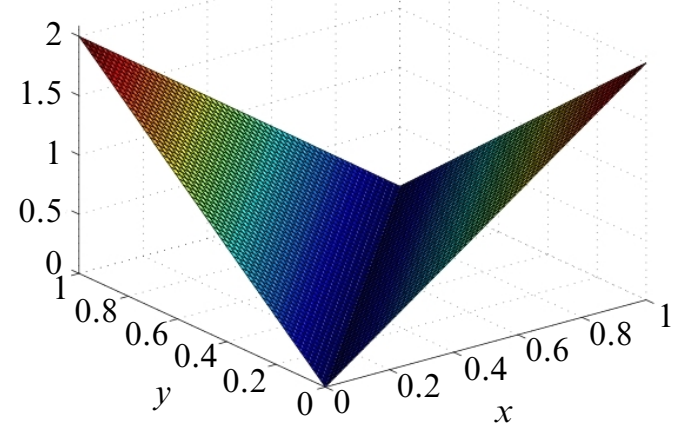

Figure 17: The metric $d$ on a diagonal 2-cell $F=\bar{e} \times \bar{e}$ with $\ell(e)=2$

\footnotetext{
${ }^{1}$ See the remarks after [5, Definition 2.1].
} 
are several cases to consider, arising from the positions of minimal-length arcs joining points of $\bar{e}$ to points of $\bar{f}$.

Case 1 Suppose that there exist vertices $v \in \partial e$ and $w \in \partial f$ such that every minimallength arc from $\bar{e}$ to $\bar{f}$ passes through $v$ and $w$; see Figure 18. Let $a:=d(\bar{e}, \bar{f})$ be the distance between $\bar{e}$ and $\bar{f}$. Without loss of generality, assume that $\partial_{1} e=v$ and

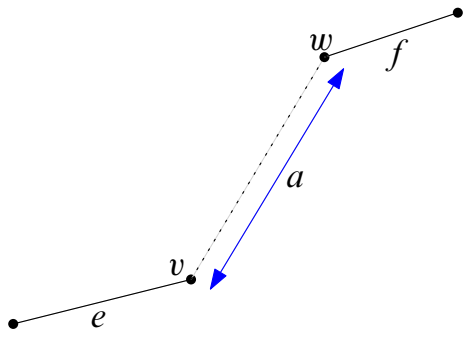

Figure 18: The situation in Case 1

$\partial_{0} f=w$. Then

$$
\begin{array}{ll}
d\left(\chi_{F}(0,0)\right)=a+\ell(e), & d\left(\chi_{F}(1,0)\right)=a, \\
d\left(\chi_{F}(0,1)\right)=a+\ell(e)+\ell(f), & d\left(\chi_{F}(1,1)\right)=a+\ell(f)
\end{array}
$$

and $d \circ \chi_{F}$ extends to an affine map $\mathbb{R}^{2} \rightarrow \mathbb{R}$; see Figure 19 and Figure 20.

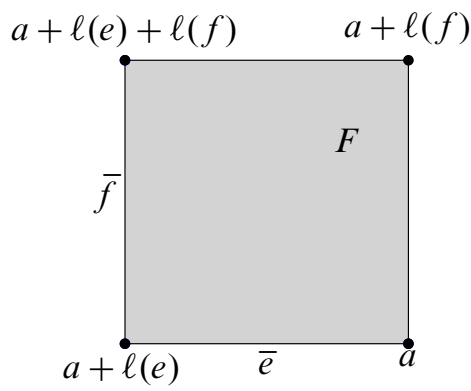

Figure 19: $d \circ \chi_{F}$ extends to an affine map $\mathbb{R}^{2} \rightarrow \mathbb{R}$

Case 2 Now suppose that we are not in Case 1. This means that for all $v \in \partial e$ and $w \in \partial f$ there is a minimal-length arc from $\bar{e}$ to $\bar{f}$ not containing $v$ or not containing $w$. We identify three subcases illustrated in Figures 21, 24 and 27.

(i) Assume that there is a vertex $v \in \partial e$ such that every minimal-length arc from $\bar{e}$ to $\bar{f}$ passes through $v$; see Figure 21 (the argument is the same if there is a vertex $w \in \partial f$ such that every minimal-length arc from $\bar{e}$ to $\bar{f}$ passes through $w$ ). Write 


$$
1-x+2 y
$$

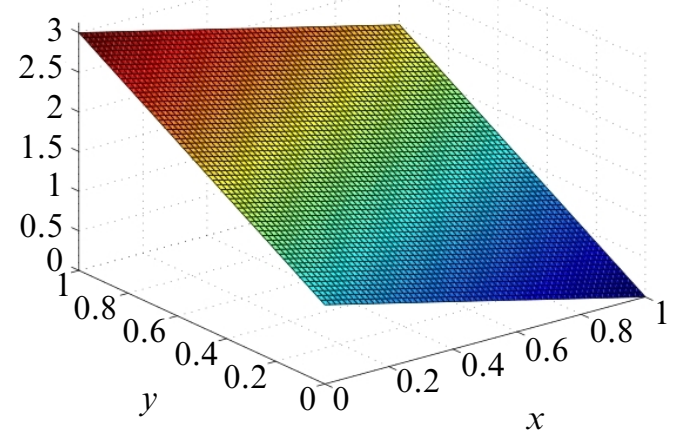

Figure 20: The graph of $d \circ \chi_{F}$ with $a=0, \ell(e)=1$ and $\ell(f)=2$

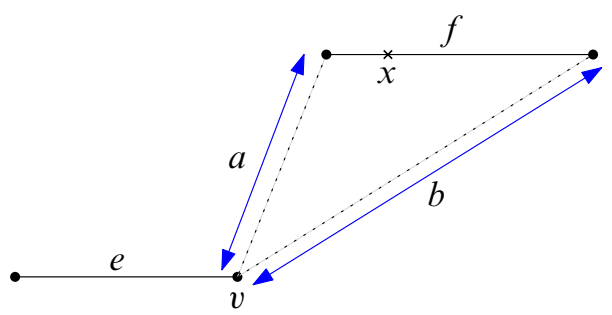

Figure 21: Case 2(i)

$a=d\left(v, \partial_{0} f\right)$ and $b=d\left(v, \partial_{1} f\right)$. By assumption, there is a point $x \in f$ such that

$$
d\left(v, \partial_{0} f\right)+d\left(\partial_{0} f, x\right)=d\left(x, \partial_{1} f\right)+d\left(\partial_{1} f, v\right) .
$$

Moreover

$$
\begin{aligned}
& d\left(\chi_{F}(0,0)\right)=a+\ell(e), \quad d\left(\chi_{F}(1,0)\right)=a, \\
& d\left(\chi_{F}(0,1)\right)=b+\ell(e), \quad d\left(\chi_{F}(1,1)\right)=b .
\end{aligned}
$$

The line $\bar{e} \times\{x\}$ separates $F$ into two closed 2 -cells $F_{1}$ and $F_{2}$. If $j \in\{1,2\}$ and $\chi_{F_{j}}: C_{F_{j}} \rightarrow F_{j}$ is a characteristic function of $F_{j}$, then $d \circ \chi_{F_{j}}$ extends to an affine $\operatorname{map} \mathbb{R}^{2} \rightarrow \mathbb{R}$; see Figures 22 and 23 .

(ii) We now assume that (i) does not hold, and that all minimal-length arcs from $\partial_{0} e$ to $\partial_{0} f$ are disjoint from all minimal-length arcs from $\partial_{1} e$ to $\partial_{1} f$. Write $a=d\left(\partial_{0} e, \partial_{0} f\right)$ and $b=d\left(\partial_{1} e, \partial_{1} f\right)$. Without loss of generality, there is a point $x \in \bar{e}$ equidistant to $\partial_{0} f$ along two distinct arcs; see Figure 24 . 


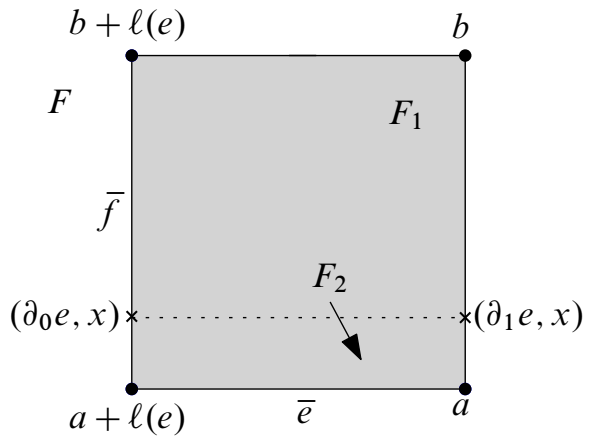

Figure 22: The separating line $\bar{e} \times\{x\}$

$$
2.5-x-|y-0.5|
$$

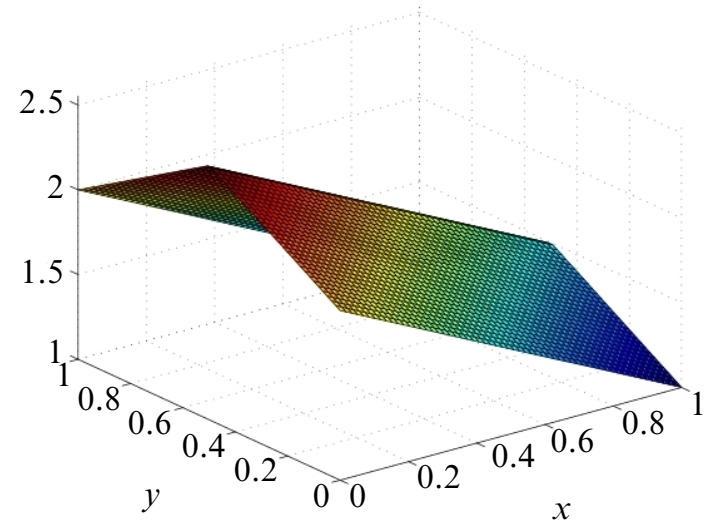

Figure 23: The graph of $d \circ \chi_{F}$ with $a=b=1$ and $\ell(e)=\ell(f)=1$

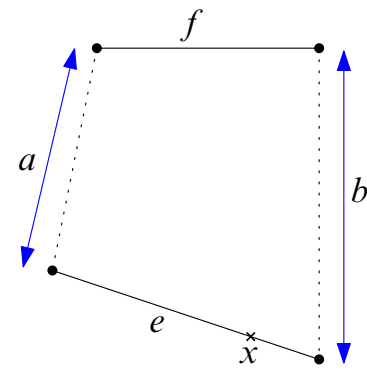

Figure 24: Case 2(ii) 
We have $d\left(\chi_{F}(0,0)\right)=a$ and $d\left(\chi_{F}(1,1)\right)=b$. There is also a diagonal line in $F$ on which $d$ is constant with value $(\ell(e)+\ell(f)+a+b) / 2$. This line separates $F$ into two closed 2-cells $F_{1}$ and $F_{2}$. If $j \in\{1,2\}$ and $\chi_{F_{j}}: C_{F_{j}} \rightarrow F_{j}$ is a characteristic function of $F_{j}$, then $d \circ \chi_{F_{j}}$ extends to an affine map $\mathbb{R}^{2} \rightarrow \mathbb{R}$; see Figures 25 and 26.

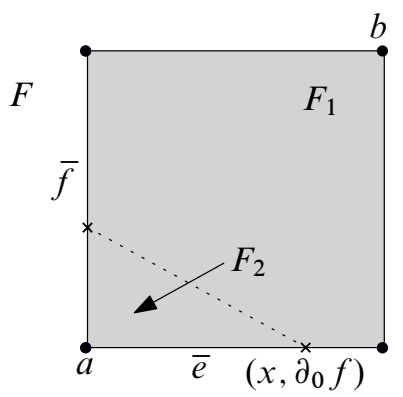

Figure 25: The separating line on which $d$ is constant

$$
2-|x+y-1|
$$

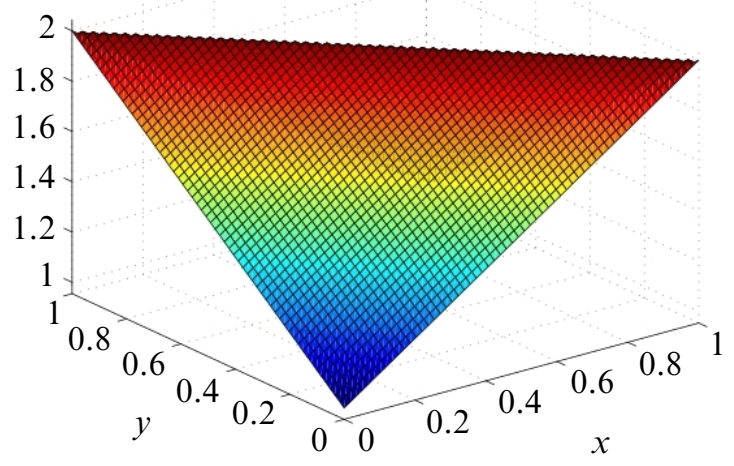

Figure 26: The graph of $d \circ \chi_{F}$ with $a=b=1$ and $\ell(e)=\ell(f)=1$; it is symmetric about $y=1-x$.

(iii) Finally, we assume that (i) does not hold and that at least one minimal-length arc from $\partial_{0} e$ to $\partial_{0} f$ intersects a minimal-length arc from $\partial_{1} e$ to $\partial_{1} f$; see Figure 27. There are points $x \in e, y \in f$ equidistant from the intersection of these arcs (otherwise we are in Case 2(i)). The lines $\{x\} \times \bar{f}$ and $\bar{e} \times\{y\}$ separate $F$ into four closed 2-cells $F_{1}, F_{2}, F_{3}, F_{4}$. If $j \in\{1,2,3,4\}$ and $\chi_{F_{j}}$ is a characteristic function of $F_{j}$, then $d \circ \chi_{F_{j}}$ extends to an affine map $\mathbb{R}^{2} \rightarrow \mathbb{R}$; see Figure 28. The graph of $d \circ \chi_{F_{j}}$ resembles the graph in Case 1 (Figure 20) for each $j \in\{1,2,3,4\}$. 


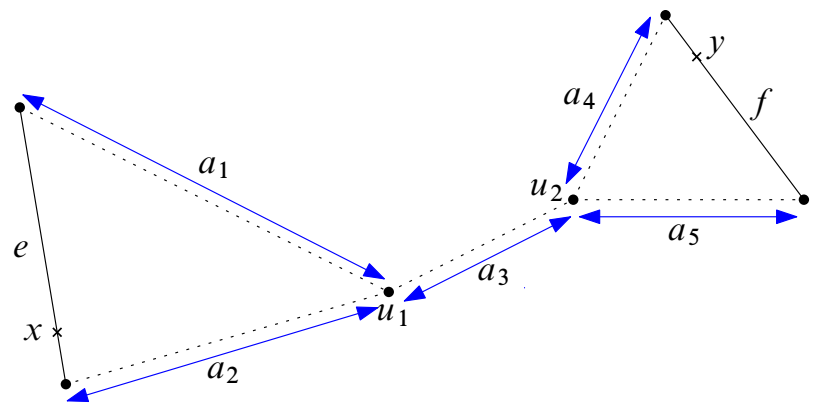

Figure 27: Case 2(iii)

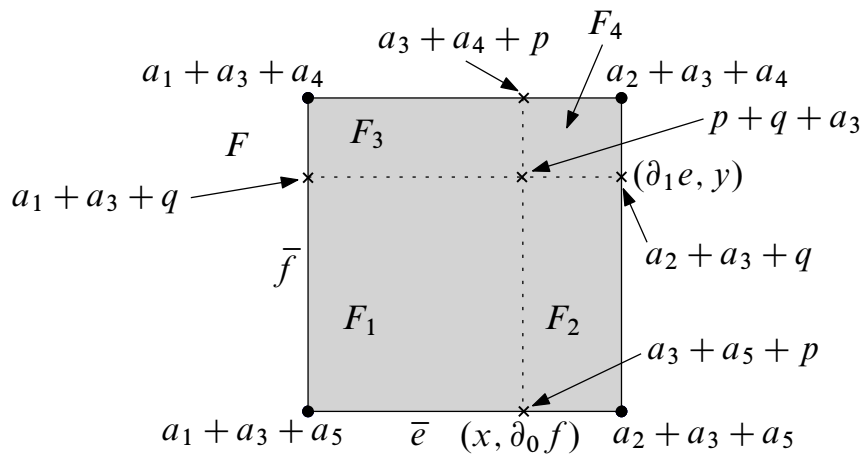

Figure 28: The graph of $d$ in Case 2(iii). Here, $p=\left(a_{1}+a_{2}+\ell(e)\right) / 2$ and $q=\left(a_{4}+a_{5}+\ell(f)\right) / 2$.

Finally, we note that under the subdivisions in Figure 16 and all subcases of Case 2 (see Figures 22, 25 and 28) $\Gamma \times \Gamma$ remains an affine polytope complex. This establishes Proposition 4.1.

\section{Critical values and homology groups}

In this section we apply PL Morse-Bott theory from Section 3 to obtain information on the homotopy type of the configuration spaces $\left\{F_{r}(\Gamma, 2)\right\}_{r>0}$. From Proposition 4.1, $d$ is an affine Morse-Bott function on $\Gamma \times \Gamma$ with respect to a suitable $\mathrm{CW}$ structure. Since $F_{r}(\Gamma, 2)=d^{-1}([2 r, \infty))$, we work with the sublevel sets of $g:=-d$, which is also an affine Morse-Bott function. 


\subsection{Critical values of the family $\left\{F_{r}(\Gamma, 2)\right\}_{r>0}$}

We refer to the introduction for the definition of regular and critical values of the family $\left\{F_{r}(\Gamma, 2)\right\}_{r>0}$ (see Definition 1.2). We write $Z(\Gamma)$ for the set of cycles in $\Gamma$ (subgraphs homeomorphic to $S^{1}$ ) and $\ell(C)$ for the length of $C \in Z(\Gamma)$. Moreover, $Z$ and $E$ denote the numbers of cycles and edges in $\Gamma$, respectively. The following theorem is the main result of this section.

Theorem 5.1 Each critical value of the family $\left\{F_{r}(\Gamma, 2)\right\}_{r>0}$ has the form

$$
\frac{1}{2}\left(\frac{1}{2} \sum_{C \in Z(\Gamma)} \varepsilon_{C} \ell(C)+\sum_{e \in E(\Gamma)} \varepsilon_{e} \ell(e)\right),
$$

where at most two of $\varepsilon_{C} \in\{0,1\}$ are nonzero and $\varepsilon_{e} \in\{0,1\}, \forall e \in E(\Gamma)$. In particular, there are fewer than $2^{E} \max \{2 Z(Z-1), 1\}$ critical values.

Proof Let $\Gamma \times \Gamma$ be equipped with the subdivision from Proposition 4.1, so that $d$ (and hence $g$ ) is an affine Morse-Bott function. Since $\Gamma \times \Gamma$ has finitely many cells, Proposition 3.19 implies that the family of sublevel sets $\left\{g^{-1}((-\infty, c])\right\}_{c \in \mathbb{R}}$ has finitely many critical values. Hence, the family $\left\{F_{r}(\Gamma, 2)\right\}_{r>0}$ has the same property.

To obtain formula (1), we refer to the proof of Proposition 4.1. Critical levels of $d$ arising from Case 1 are contained in the set $\{d(v, w)\}_{v, w \in V(\Gamma)}$ of distances between vertices. Such distances have the form $\sum_{e \in E(\Gamma)} \varepsilon_{e} \ell(e)$ for some coefficients $\varepsilon_{e} \in\{0,1\}$.

Case 2(i) contributes critical levels of the form

$$
\varepsilon_{1} \ell(e)+\varepsilon_{2} \ell(C),
$$

for some edge $e$, cycle $C$ and coefficients $\varepsilon_{1}, \varepsilon_{2} \in\{0,1\}$, the latter not both zero. Critical levels of $d$ arising from Case 2(ii) have the form $\ell(C) / 2$ for some $C \in Z(\Gamma)$. Case 2(iii) contributes critical levels of the form

$$
\frac{1}{2}\left(\varepsilon_{1} \ell\left(C_{1}\right)+\varepsilon_{2} \ell\left(C_{2}\right)\right)+\sum_{e \in E(\Gamma)} \varepsilon_{e} \ell(e)
$$

for some coefficients $\varepsilon_{1}, \varepsilon_{2}, \varepsilon_{e} \in\{0,1\}$, not all zero; see Figure 28. Finally, we combine these observations to obtain formula (1). The factor of $1 / 2$ appears because a critical value of the family $\left\{F_{r}(\Gamma, 2)\right\}_{r>0}$ is a critical level of $d$ divided by two.

Example 5.2 Let $\Gamma$ be the $Y$-graph from Example 2.8. Excluding numbers exceeding $\frac{1}{2}$ diam $\Gamma$, formula (1) yields two possible critical values of the family $\left\{F_{r}(\Gamma, 2)\right\}_{r>0}$, namely $1 / 2$ and 1 . It is easy to compute that $F_{r}(\Gamma, 2) \simeq S^{1}$ for $r \in(0,1 / 2]$, 
$F_{r}(\Gamma, 2) \simeq Q_{6}$ for $r \in(1 / 2,1]$ and $F_{r}(\Gamma, 2)=\varnothing$ for all $r>1$ (for example, start with [11, Lemma 1.1]). Hence the number of possible critical values given by (1) is exact for this graph.

\subsection{Behaviour across a critical value}

Suppose that $0<a<D / 2<b$ are such that $D$ is the only critical level of $d$ in $[2 a, 2 b]$. We apply the results of Section 3.3 to compute rk $H_{k}\left(F_{a}(\Gamma, 2), F_{b}(\Gamma, 2) ; \mathbb{Q}\right)$ for $k=0,1,2$.

The critical subcomplex, descending sets and descending links Since there are no critical cells of dimension two, the critical subcomplex $C(d)$ is a graph. In particular, $C(d, D)$ is a graph. Let $Z$ be a component of $C(d, D)$. From the previous section (see Figures 22, 25 and 28) only the 0 -cells of $Z$ need be considered to determine $\operatorname{Lk}_{\downarrow}(Z)$, via the disjoint union

$$
\operatorname{Lk}_{\downarrow}(Z)=\coprod_{(v, w) \in Z^{(0)}} \operatorname{Lk}_{\downarrow}(v, w) .
$$

Let $(v, w)$ be a 0 -cell of $Z$. This means that $(v, w)$ is a vertex of $\Gamma \times \Gamma$ with respect to the subdivision from Proposition 4.1. The $g$-descending cells $F$ such that $\left.g\right|_{F}$ achieves its maximum on $(v, w)$ are found as follows.

Case 1 Suppose that both $v$ and $w$ are vertices of $\Gamma$. Let $e_{1}, \ldots, e_{p}$ be the edges incident to $v$ such that $y \mapsto d(y, w)$ increases on $e_{i}$ as we move away from $v$. Let $f_{1}, \ldots, f_{q}$ be the edges incident to $w$ such that $y \mapsto d(y, v)$ increases on $f_{j}$ as we move away from $w$; see Figure 29. Then the above cells $F$ are the largest cells of $\Gamma \times \Gamma$ containing $(v, w)$ and contained in the products $\bar{e}_{i} \times \bar{f}_{j}$. The subset $\operatorname{Lk}_{\downarrow}(v, w) \cap F$ of $\operatorname{Lk}_{\downarrow}(v, w)$ contained in $F$ is shown in Figure 30. It follows that $\operatorname{Lk}_{\downarrow}(v, w)=K_{p, q}$

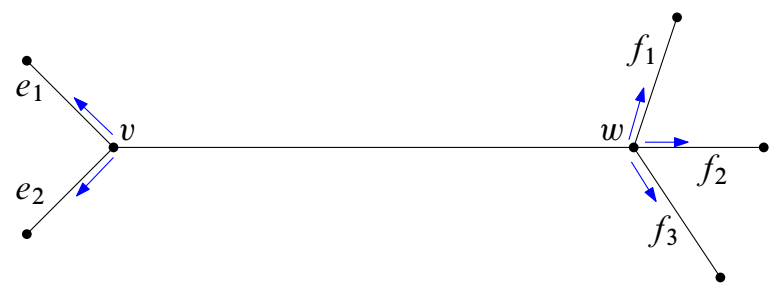

Figure 29: Finding the $g$-descending cells incident to $(v, w)$

(the complete bipartite graph of type $(p, q)$ ), where we set $K_{0, m}:=Q_{m}$ to be a discrete set of $m \geq 1$ points and $K_{0,0}:=\varnothing$. 

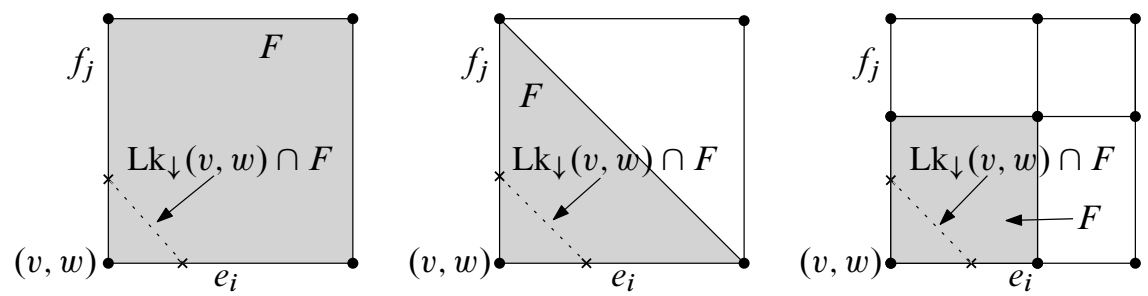

Figure 30: $g$-Descending cells $F$ incident to $(v, w)$, with $\operatorname{Lk}_{\downarrow}(v, w) \cap F$ shown in each case

Case 2 Now suppose that one of $v, w$ is a vertex of $\Gamma$ and the other is equidistant (at distance $D$ ) along at least two distinct paths; see Figure 31 . Let $e_{1}, \ldots, e_{m}$ be the

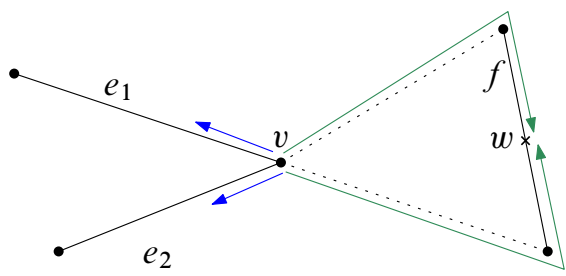

Figure 31: The situation in Case 2

edges incident to $v$ satisfying the same property as in Case 1 above. Then the cells $F$ such that $\left.g\right|_{F}$ achieves its maximum on $(v, w)$ are precisely $\bar{e}_{i} \times\{w\}$, for $1 \leq i \leq m$. Hence $\operatorname{Lk}_{\downarrow}(v, w)=Q_{m}$ (a discrete set of $m$ points). If $v, w$ are both not vertices of $\Gamma$, then $(v, w)$ is a vertex of the subdivision of $\Gamma \times \Gamma$ from Case 2(iii) in Section 4; see Figure 28. In this case $\operatorname{Lk}_{\downarrow}(v, w)=\varnothing$, since $(v, w)$ is a local maximum of $d$.

The map $h: \operatorname{Lk}_{\downarrow}(Z) \rightarrow Z \quad$ It is easy to determine the map $h: \operatorname{Lk}_{\downarrow}(Z) \rightarrow Z$ for $Z$ a component of $C(d, D)$. Indeed, $\operatorname{Lk}_{\downarrow}(Z)$ is the disjoint union $\bigsqcup_{(v, w) \in Z^{(0)}} \operatorname{Lk}_{\downarrow}(v, w)$ and for each $(v, w) \in Z^{(0)},\left.h\right|_{\operatorname{Lk}_{\downarrow}(v, w)}$ is constant with value $(v, w)$. In particular, for $j \geq 0$,

$$
\operatorname{ker}\left(h_{*}: H_{j}\left(\operatorname{Lk}_{\downarrow}(Z)\right) \rightarrow H_{j}(Z)\right)=H_{j}\left(\operatorname{Lk}_{\downarrow}(Z)\right)=\bigoplus_{(v, w) \in Z^{(0)}} H_{j}\left(\operatorname{Lk}_{\downarrow}(v, w)\right),
$$

$\operatorname{coker}\left(h_{*}: H_{j}\left(\operatorname{Lk}_{\downarrow}(Z)\right) \rightarrow H_{j}(Z)\right)=H_{j}(Z)$. 
From above, $\operatorname{Lk}_{\downarrow}(v, w)=K_{p, q}$, where $p=p(v, w), q=q(v, w) \geq 0$ are as previously defined. Thus,

$$
\begin{aligned}
& H_{j}\left(K_{p, q}\right)= \begin{cases}\mathbb{Z} & j=0, \\
\mathbb{Z}^{(p-1)(q-1)} & j=1, \quad \text { for } p, q \geq 1, \\
0 & j \geq 2,\end{cases} \\
& H_{j}\left(K_{0, n}\right)=\left\{\begin{array}{ll}
\mathbb{Z}^{n} & j=0, \\
0 & j \geq 1,
\end{array} \quad \text { for } n \geq 0 .\right.
\end{aligned}
$$

Applying the main result Let $Z_{1}, \ldots, Z_{n}$ be the components of $C(d, D)$. Applying a corollary of Proposition 3.21 (see Remark 3.26(ii)) gives the following statement.

Theorem 5.3 For each $j \geq 0$,

$$
\begin{aligned}
\operatorname{rk} H_{j}\left(F_{a}, F_{b} ; \mathbb{Q}\right)=\sum_{i=1}^{n}(\operatorname{rk} \operatorname{coker}( & \left.\left(h_{i}\right)_{*}: H_{j}\left(\operatorname{Lk}_{\downarrow}\left(Z_{i}\right)\right) \rightarrow H_{j}\left(Z_{i}\right)\right) \\
& \left.+\operatorname{rk} \operatorname{ker}\left(\left(h_{i}\right)_{*}: H_{j-1}\left(\operatorname{Lk}_{\downarrow}\left(Z_{i}\right)\right) \rightarrow H_{j-1}\left(Z_{i}\right)\right)\right) .
\end{aligned}
$$

We now analyse this statement for $j=0,1,2$ separately. When $j=0$, we have

$$
\operatorname{rk} \operatorname{coker}\left(\left(h_{i}\right)_{*}: H_{0}\left(\operatorname{Lk}_{\downarrow}\left(Z_{i}\right)\right) \rightarrow H_{0}\left(Z_{i}\right)\right)=\operatorname{rk} H_{0}\left(Z_{i}\right)=1 \text {, }
$$

since $\left(h_{i}\right)_{*}=0$ and $Z_{i}$ is connected. Hence we find that $\mathrm{rk} H_{0}\left(F_{a}, F_{b}\right)$ is the number of path components of $C(d, D)$.

For $j=1$, we have

$$
\begin{aligned}
\operatorname{rkcoker}\left(\left(h_{i}\right)_{*}: H_{1}\left(\operatorname{Lk}_{\downarrow}\left(Z_{i}\right)\right) \rightarrow\right. & \left.H_{1}\left(Z_{i}\right)\right)+\operatorname{rk} \operatorname{ker}\left(\left(h_{i}\right)_{*}: H_{0}\left(\operatorname{Lk}_{\downarrow}\left(Z_{i}\right)\right) \rightarrow H_{0}\left(Z_{i}\right)\right) \\
& =\operatorname{rk} H_{1}\left(Z_{i}\right)+\operatorname{rk} H_{0}\left(\operatorname{Lk}_{\downarrow}\left(Z_{i}\right)\right) \quad\left(\text { since }\left(h_{i}\right)_{*}=0\right) \\
& =\operatorname{rk} H_{1}\left(Z_{i}\right)+\sum_{(v, w) \in Z_{i}^{(0)}} \operatorname{rk} H_{0}\left(\operatorname{Lk}_{\downarrow}(v, w)\right) .
\end{aligned}
$$

For each $(v, w) \in Z_{i}^{(0)}, \operatorname{rk}_{0}\left(\operatorname{Lk}_{\downarrow}(v, w)\right)$ may be computed explicitly from formulas (2) and (3). Moreover, $Z_{i}$ is a connected graph, so we have an algorithm for computing rk $H_{1}\left(Z_{i}\right)$ and thus also for computing rk $H_{1}\left(F_{a}, F_{b}\right)$. 
For $j=2$, we use the facts that $H_{2}\left(Z_{i}\right)=0$ and $\left(h_{i}\right)_{*}=0$ to obtain $\operatorname{rkcoker}\left(\left(h_{i}\right)_{*}: H_{2}\left(\operatorname{Lk}_{\downarrow}\left(Z_{i}\right)\right) \rightarrow H_{2}\left(Z_{i}\right)\right)+\operatorname{rk} \operatorname{ker}\left(\left(h_{i}\right)_{*}: H_{1}\left(\operatorname{Lk}_{\downarrow}\left(Z_{i}\right)\right) \rightarrow H_{1}\left(Z_{i}\right)\right)$

$$
=0+\operatorname{rk} H_{1}\left(\operatorname{Lk}_{\downarrow}\left(Z_{i}\right)\right)=\sum_{(v, w) \in Z_{i}^{(0)}} \operatorname{rk} H_{1}\left(\operatorname{Lk}_{\downarrow}(v, w)\right)
$$

$$
=\sum_{\substack{(v, w) \in Z_{i}^{(0)} \\ p(v, w), q(v, w) \geq 2}}(p(v, w)-1)(q(v, w)-1) .
$$

Finally, we describe explicit generators for $H_{2}\left(F_{a}, F_{b}\right)$. Suppose that $(v, w) \in Z_{i}^{(0)}$ contributes to the sum (4). We have $p(v, w), q(v, w) \geq 2$; see Figure 32. The

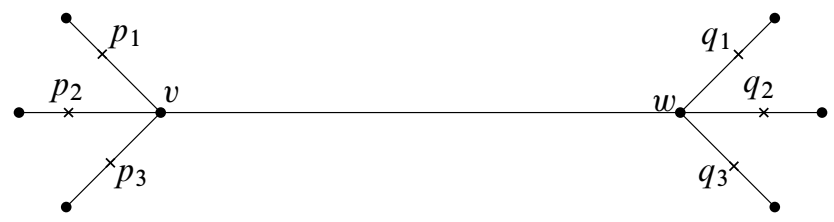

Figure 32: $p(v, w), q(v, w) \geq 2$

$g$-descending link of $(v, w)$ is $\operatorname{Lk}_{\downarrow}(v, w)=K_{p, q}$; see Figure 33. Choose the maximal
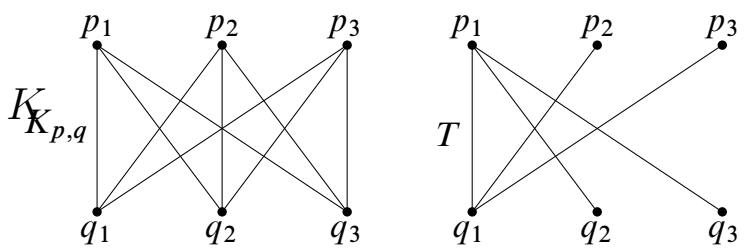

Figure 33: The descending link $\operatorname{Lk}_{\downarrow}(v, w)=K_{p, q}$ and the maximal tree $T$

tree $T$ in $K_{p, q}$ as shown in Figure 33. There are $(p-1)(q-1)$ edges of $K_{p, q}$ not contained in $T$. The generators of $H_{1}\left(K_{p, q}\right)$ are the cycles in $K_{p, q}$ defined by the sequences $\left(p_{1}, q_{1}, p_{i}, q_{j}, p_{1}\right)$ for $i, j \geq 2$. From the exact sequence of the pair $\left(F_{a}, F_{b}\right)$, $H_{2}\left(F_{a}, F_{b}\right)$ is isomorphic to a subgroup of $H_{1}\left(F_{b}\right)$. We interpret the generators of $H_{1}\left(K_{p, q}\right)$ as generators of $H_{1}\left(F_{b}\right)$ which lie in the kernel of $H_{1}\left(F_{b}\right) \rightarrow H_{1}\left(F_{a}\right)$; see Figure 34 .

\section{The case $n>2$ and open problems}

In this section we discuss configuration spaces of $n>2$ thick particles on a graph and mention some open problems. For $n>2$ and $r>0$, the configuration space of $n$ thick 


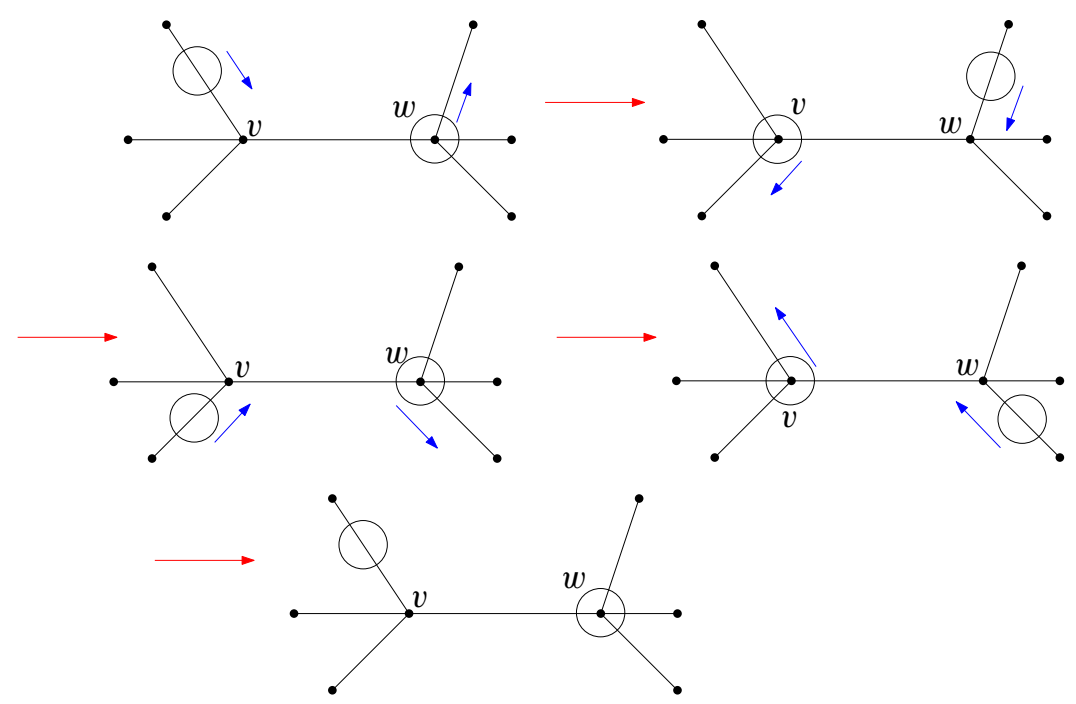

Figure 34: Generators of $H_{1}\left(K_{p, q}\right)$ interpreted as cycles in $H_{1}\left(F_{b}\right)$

particles on $\Gamma$ is

$$
F_{r}(\Gamma, n):=\left\{\left(x_{1}, \ldots, x_{n}\right) \in \Gamma^{n}: \min _{i<j}\left\{d\left(x_{i}, x_{j}\right)\right\} \geq 2 r\right\},
$$

topologised as a subspace of the product $\Gamma^{n}$. From the viewpoint of topological robotics $F_{r}(\Gamma, n)$ models the collision-free motion of $n$ robots of radius $r$ on $\Gamma$, permitting tangencies between robots. In [7] we apply the PL Morse-Bott theory developed in Section 3 to the family of configuration spaces $\left\{F_{r}(\Gamma, n)\right\}_{r>0}$. We obtain a result analogous to Theorem 5.1 (see [7, Section 6.1]):

Theorem 6.1 The family $\left\{F_{r}(\Gamma, n)\right\}_{r>0}$ has finitely many critical values.

In [7, Section 6.2] we also describe these critical values explicitly in terms of the metric data in the special case when $\Gamma$ is a tree. However, there are many interesting open questions arising from the study of configuration spaces of thick particles, some of which we now list. I thank the referee for suggesting problems (1)-(4).

\section{Open problems}

(1) For every presented group $G$ is there a graph $\Gamma$ and an $r>0$ such that $\pi_{1}\left(F_{r}(\Gamma, 2)\right)=G$ ?

(2) Is the bound from Theorem 5.1 sharp for certain families of graphs? In particular, does the number of critical values grow exponentially in the number of edges? 
(3) A critical value is necessary for a change in homotopy type. Is there a simple sufficient condition?

(4) For $n>2$, provide an upper bound on $b_{0}\left(F_{r}(\Gamma, n)\right)$ in terms of $\Gamma, n$ and $r$. Develop an algorithm for computing $b_{0}\left(F_{r}(\Gamma, n)\right)$.

(5) Given a general noncontractible graph $\Gamma$, express the critical values of the family $\left\{F_{r}(\Gamma, n)\right\}_{r>0}$ in terms of metric properties of $\Gamma$.

(6) Using the PL Morse-Bott theory developed in Section 3 or otherwise, investigate the topology of other thick particle configuration spaces of practical significance. For example, choose a subgraph $K \subset \Gamma$ and consider the subspace of $F_{r}(\Gamma, n)$ comprising the configurations $\left(x_{1}, \ldots, x_{n}\right)$ such that at most $k$ points $x_{1}, \ldots, x_{n}$ lie in $K$ :

$$
\left\{\left(x_{1}, \ldots, x_{n}\right) \in \Gamma^{n}: \min _{i<j}\left\{d\left(x_{i}, x_{j}\right)\right\} \geq 2 r,\left|\left\{x_{1}, \ldots, x_{n}\right\} \cap K\right| \leq k\right\} .
$$

This space may be useful in applied contexts as it models the collision-free motion of $n$ robots of radius $r$ on $\Gamma$ such that at most $k$ robots are permitted in the subgraph $K$.

\section{References}

[1] A Abrams, Configuration spaces and braid groups of graphs, $\mathrm{PhD}$ thesis, University of California, Berkeley (2000) Available at http://www.mathcs.emory.edu/ abrams/publications/thesis.ps

[2] A Abrams, R Ghrist, Finding topology in a factory: configuration spaces, Amer. Math. Monthly 109 (2002) 140-150 MR1903151

[3] K Barnett, The configuration space of two particles moving on a graph, $\mathrm{PhD}$ thesis, Durham University (2010) Available at http://etheses.dur.ac.uk

[4] K Barnett, M Farber, Topology of configuration space of two particles on a graph. I, Algebr. Geom. Topol. 9 (2009) 593-624 MR2491587

[5] M Bestvina, PL Morse theory, Math. Commun. 13 (2008) 149-162 MR2488666

[6] M Bestvina, N Brady, Morse theory and finiteness properties of groups, Invent. Math. 129 (1997) 445-470 MR1465330

[7] K Deeley, Configuration spaces of thick particles on graphs, $\mathrm{PhD}$ thesis, Durham University (2011) Available at http://etheses.dur.ac.uk

[8] M Farber, Collision free motion planning on graphs, from: "Algorithmic foundations of robotics IV", (M Erdmann, D Hsu, M Overmars, A F van der Stappen, editors), Springer Tracts in Adv. Robotics 17, Springer, Berlin (2005) 123-138 
[9] M Farber, Invitation to topological robotics, Zürich Lectures in Advanced Math., Eur. Math. Soc., Zürich (2008) MR2455573

[10] M Farber, E Hanbury, Topology of configuration space of two particles on a graph, II, Algebr. Geom. Topol. 10 (2010) 2203-2227 MR2745669

[11] R Ghrist, Configuration spaces and braid groups on graphs in robotics, from: "Knots, braids, and mapping class groups-papers dedicated to Joan S Birman (New York, 1998)", (J Gilman, W W Menasco, X-S Lin, editors), AMS/IP Stud. Adv. Math. 24, Amer. Math. Soc. (2001) 29-40 MR1873106

[12] R Ghrist, Configuration spaces, braids, and robotics, from: "Braids", (A J Berrick, F R Cohen, E Hanbury, Y-L Wong, J Wu, editors), Lect. Notes Ser. Inst. Math. Sci. Natl. Univ. Singap. 19, World Sci. Publ., Hackensack, NJ (2010) 263-304 MR2605308

[13] R Ghrist, D E Koditschek, Safe cooperative robotic motions via dynamics on graphs, from: "Eighth Intl. Symp. on Robotic Research", (Y Nakayama, editor), SpringerVerlag, Berlin (1998) 81-92

[14] R Ghrist, D E Koditschek, Safe cooperative robot dynamics on graphs, SIAM J. Control Optim. 40 (2002) 1556-1575 MR1882808

[15] K H Ko, H W Park, Characteristics of graph braid groups arXiv: 1101.2648v1

[16] J Świątkowski, Estimates for homological dimension of configuration spaces of graphs, Colloq. Math. 89 (2001) 69-79 MR1853416

Department of Mathematical Sciences, Durham University

Durham DH1 3LE, UK

kenneth.deeley@durham.ac.uk

Received: 23 October $2010 \quad$ Revised: 15 March 2011 